\title{
Evaluation of a source-function wavemaker for generating random directionally spread waves in the sea-swell band
}

\author{
S.H. Suanda ${ }^{\mathrm{a}, *}$, S. Perez ${ }^{\mathrm{a}}$, F. Feddersen ${ }^{\mathrm{a}}$ \\ ${ }^{a}$ Scripps Institution of Oceanography
}

\begin{abstract}
A source-function wavemaker for wave-resolving models is evaluated for its capability to reproduce random directionally spread wave fields in the sea-swell band $(0.04-0.3 \mathrm{~Hz})$ relevant for realistic nearshore applications. The wavemaker is tested with a range of input wave characteristics defined by the non-dimensional amplitude $(a / h)$, wavenumber $(k h)$, wavemaker width, mean wave angle and directional spread. The $a / h$ and $k h$ dependency of modeled results are collapsed with the Ursell number $\left(\mathrm{Ur}=(a / h) /(k h)^{2}\right)$. For monochromatic waves, the wavemaker accurately reproduced the input wave height for $\mathrm{Ur}<1$, with no dependence on nondimensional wavemaker width. For random uni-directional waves, the wavemaker simulated well a Pierson-Moskowitz input spectrum. Frequency-integrated statistics are also reproduced with less than $2 \%$ difference between modeled to input significant wave height and $<10 \%$ difference between modeled to input mean frequency for $\mathrm{Ur}<0.2$. For random directionally spread waves, the wavemaker reproduced input frequency-dependent and bulk mean wave angle and directional spread to within $4^{\circ}$ at $\mathrm{Ur}<0.12$. Lastly, the wavemaker simulated well the spectra, mean wave angle, and directional spread of a bimodal wave field with opposing sea and swell. Based on the Ur $<0.12$ constraint, a range of dimensional wave height, period, and depth constraints are explored for realistic sea-swell band field application. The wavemaker's ability to generate waves that match the input statistical properties commonly derived from field measurements demonstrates that it can be used effectively in a range of nearshore science and engineering applications.
\end{abstract}

\section{Introduction}

Wave-resolving numerical models have a variety of oceanographic and engineering application including simulating nearshore wave transformation (e.g., Nwogu, 1993; Wei and Kirby, 1995; Gobbi et al., 2000; Madsen et al., 2003; Kirby, 2003; Torres-Freyermuth et al., 2010, Lara et al., 2011; Shi et al., 2012, Higuera et al., 2015), and the resulting wave-driven circulation (e.g., Chen et al., 1999, 2003). Boussinesq models (a class of wave-resolving models) have

\footnotetext{
* Corresponding author

Email address: ssuanda@ucsd.edu (S.H. Suanda)
} 
been used in field-scale studies of nearshore processes with random waves including the study of surfzone vorticity and transient rip currents (e.g., Johnson and Pattiaratchi, 2006, Feddersen, 2014; Suanda and Feddersen, 2015), surfzone drifter (Spydell and Feddersen, 2009), and dye (Feddersen et al., 2011; Clark et al., 2011) dispersion. In these studies, the model must accurately reproduce the requested random directionally spread wave field in the sea-swell band $(0.04-0.3 \mathrm{~Hz})$ to generate the appropriate energy fluxes, Stokes drift, or radiation stresses that drive nearshore processes.

Boussinesq models essentially include nonlinear and dispersive effects into the shallow water equations by perturbation expansion (e.g., Peregrine, 1967). Nonlinearity is represented by the nondimensional parameter $a / h$, the ratio of wave amplitude to water depth, and dispersion is represented by the nondimensional parameter $k h$, where $k$ is the wavenumber. These two parameters can be combined to form the Ursell number,

$$
\mathrm{Ur}=\frac{a / h}{(k h)^{2}},
$$

a metric of long-wave nonlinearity, which can be up to $O(1)$. The extended Boussinesq equations allow improved wave dispersion (and wave celerity) to $k h \approx 2$, consistent with intermediate-depth water applications (e.g., Nwogu, 1993; Gobbi et al., 2000; Madsen et al. 2002, 2003). For field-scale studies, waves seaward of the surfzone generally have small $a / h$ as breaking can occur at $a / h>0.2$.

Three methods are used to generate the incident wave field in wave-resolving numerical models. The first is to prescribe incident waves at a static offshore boundary, which although computationally efficient, has issues with partially-reflected outgoing waves resulting in steady energy increases within the domain (e.g., Wei et al., 1999; Higuera et al., 2013). Partial reflections are overcome by active wave absorption techniques (e.g., Schäffer and Klopman, 2000), which have been applied to wave generation in Reynolds-Averaged Navier-Stokes models (RANS) (e.g., Torres-Freyermuth et al., 2010, Higuera et al., 2013). Another wave generation and active absorption method is to use moving boundaries (virtual paddles) within a model (e.g., Lara et al., 2011; Cao and Wan, 2014; Higuera et al., 2015). This method accurately generates unidirectional wave spectra including bound long-waves in laboratory studies (Lara et al., 2011).

A third method is to embed the wavemaker within the model domain with the addition of sponge layers for wave damping at the boundaries (e.g., Larsen and Dancy, 1983; Wei et al. 1999; Lin and Liu, 1999; Lara et al., 2006). The method has been used in both Boussinesqtype (e.g., Wei et al., 1999; Schäffer and Sørensen, 2006; Kim et al., 2007; Liam et al., 2014) and RANS models (e.g., Lin and Liu, 1999; Hafsia et al., 2009; Perić and Abdel-Maksoud, 
2015) and avoids the complication of both generating and absorbing waves at a boundary. Internal wavemakers first generated waves on a single line (cross-shore delta function) source (Larsen and Dancy, 1983), which continues to be used in Boussinesq models (e.g., Schäffer and Sørensen, 2006; Kim et al., 2007). Wei et al. (1999) (hereafter W99) developed a mass source function wavemaker on an alongshore strip with cross-shore width $W$ from a Green's function solution to the linearized extended Boussinesq equations of $N w o g u(1993)$. This wavemaker width $W$ introduces another non-dimensional parameter $\delta \propto W / \lambda$ where $\lambda$ is a characteristic wavelength associated with the wave cyclic frequency $f$ through the linear dispersion relation. For Boussinesq models, all internal wavemakers are based on linearized model equations (e.g., Wei et al., 1999; Schäffer and Sørensen, 2006; Kim et al., 2007, Liam et al., 2014), and is acknowledged to result in errors for highly nonlinear waves. The source function method continues to be improved upon. For example, extensions have been developed for wave generation in currents (Chawla and Kirby, 2000). Generation of highly nonlinear waves is improved with an adjustment zone where wave model nonlinearity gradually grows (Liam et al., 2014 ).

To simulate realistic nearshore conditions, a wavemaker must be tested to determine its accuracy in generating the particular user-specified wave field. The W99 wavemaker has been tested over a limited nondimensional parameter $(a / h, k h, \delta)$ range relevant to nearshore studies. Using $\delta=0.3$, the wavemaker accurately generated one-dimensional (1D) monochromatic waves for $4 k h$ (spanning 0.8-8) and $3 a / h$ (spanning 0.05-0.15) using both the linearized and nonlinear extended Bousinessq equations (W99). Two-dimensional input and model phase comparison tests have shown good performance for waves propagating over a shoal (e.g., Wei et al., 1999; Liam et al., 2014). W99 also showed good phase comparison for 1D random wave cases at peak $k h$ of 1.0 and 2.1 and average $a / h \approx 0.04$.

Although, fixed (e.g., Torres-Freyermuth et al., 2010) and moving boundary methods (e.g., Lara et al., 2011) have been tested with laboratory studies, most comparisons between modeled and input frequency-directional spectra for random directionally-spread wave fields focus on a single case (e.g., Wei et al., 1999; Higuera et al., 2013). While in some cases the full directional wave spectrum is known a priori, usually only frequency spectra and directional moments (e.g., Kuik et al., 1988), derivable from a wave buoy or co-located pressure sensor and current meter (known as PUV) are known. In realistic random directional wave fields, two additional frequency dependent parameters are introduced; the mean wave angle $\theta_{2}(f)$ and directional spread $\sigma_{\theta}(f)$ Kuik et al., 1988). Bulk (energy-weighted) mean wave angle $\bar{\theta}_{2}$ and directional spread $\bar{\sigma}_{\theta}$ (see Appendix) can also be used to characterize the wave field. No studies have examined reproducing frequency-dependent directional moments (Kuik et al., 1988). 
The source function wavemaker largely reproduced a single input two-dimensional (2D) random wave field with $a / h=0.06$, peak $k h=1.3$, normally incident waves with bulk mean wave angle $\bar{\theta}_{2}=0^{\circ}$, and wave directional spread $\bar{\sigma}_{\theta}=10^{\circ}$ (W99). However, the ratio of modeled to input significant wave height $H_{\mathrm{s}}^{(\mathrm{m})} / H_{\mathrm{s}}^{(\mathrm{i})}=0.93$ and the modeled mean wave angle deviated from the input. The W99 wavemaker accurately generated $H_{\mathrm{s}}$ for a single input directional wave spectrum at Duck NC, but other wave statistics were not tested (Chen et al. 2003). In addition, the W99 wavemaker generated the observed spectra $S_{\eta \eta}(f)$, mean wave angle $\theta_{2}(f)$, and directional spread $\sigma_{\theta}(f)$ in the sea-swell band for 5 field cases at Huntington Beach, CA reasonably well (Feddersen et al., 2011). Aside from these examples, the W99 wavemaker remains to be tested over a $\left(\delta, a / h, k h, \bar{\theta}_{2}, \bar{\sigma}_{\theta}\right)$ parameter range appropriate to field-scale studies.

A tested Boussinesq model wavemaker that can generate a requested random directionallyspread wave field in the sea-swell band is needed for realistic field-scale nearshore science and engineering application. Here, the W99 wavemaker, implemented within the nonlinear extended Boussinesq model funwaveC (Feddersen et al., 2011), is evaluated for it's ability to accurately generate sea-swell band random directionally-spread wave fields across a parameter space relevant to realistic nearshore environments. The evaluation is made with comparison of frequency-dependent wave spectra and directional wave moments. In section 2, the W99 wavemaker and its application to generating monochromatic and random waves is presented. The set up and parameter space for a sequence of monochromatic, random uni-directional, and random directionally spread wave cases are described in Section 3. Section 4 presents various results comparing input to modeled wave properties across the tests. Section 5 and 6 provide a discussion and summary, respectively.

\section{Wei et al. (1999) wavemaker description}

\subsection{Background}

The Boussinesq wave model domain is rectangular with cross-shore coordinate $x$, alongshore coordinate $y$, and a flat bottom of depth $h$. The model has cross-shore domain width $L_{x}$ and alongshore domain width $L_{y}$, with alongshore periodic boundary conditions. The wavemaker is implemented as an alongshore strip with cross-shore width $W$ away from the onshore and offshore domain boundaries where sponge layers are applied to absorb outgoing wave energy (e.g., Fig. 19) (Larsen and Dancy, 1983; Wei et al., 1999). The wavemaker formulation can be applied to any Boussinesq equations. Here, the extended Boussinesq equations of Nwogu (1993) are used. These equations include weak nonlinearity and higher-order dispersion accurate to $k h \approx 2$ (Gobbi et al. , 2000). In the Boussinesq mass conservation equation the W99 
source function wavemaker has the form of

$$
\frac{\partial \eta}{\partial t}+\ldots=f(x, y, t)
$$

where $t$ is time, $\eta$ is the free surface, and $f(x, y, t)$ represents the wavemaker mass source. W99 developed a wavemaker forcing $f(x, y, t)$ separable in $x$ and $(y, t)$,

$$
f(x, y, t)=G\left(x-x_{\mathrm{WM}}\right) F(y, t)
$$

where $x_{\mathrm{WM}}$ is the center location of the wavemaker. The cross-shore wavemaker structure $G\left(x-x_{\mathrm{WM}}\right)$ is non-zero over a finite wavemaker width (indicated by dark gray shading in Fig. 19,b)

$$
W=\frac{1}{2} \delta \lambda
$$

which depends on a characteristic wavelength $\lambda$ and the nondimensional wavemaker width $\delta$. Defining $x^{\prime}=x-x_{\mathrm{WM}}$, the W99 form for $G\left(x^{\prime}\right)$ is the smooth shape

$$
G\left(x^{\prime}\right)=\exp \left(-\beta x^{2}\right)
$$

where

$$
\beta=80(\delta \lambda)^{-2}
$$

so that $G(W / 2)<0.01$ is a small number. W99 used a nondimensional wavemaker width $\delta$ between 0.3-0.5, whereas Larsen and Dancy (1983) generated waves at a single grid point with $\delta \rightarrow 0$. The effect of $\delta$ is discussed in Section 4 .

\subsection{Monochromatic Waves}

Monochromatic waves (i.e., a single-frequency, long-crested wave) propagating at an angle $\theta$ to the $+x$ direction are described by an amplitude $a$ and radian frequency $\omega(=2 \pi f)$,

$$
\eta=a \cos \left(k_{x} x+k_{y} y-\omega t\right)
$$

where the vector wavenumber components are $k_{x}=k \cos (\theta), k_{y}=k \sin (\theta)$. The radian frequency is related to wavenumber $k$ through the linear dispersion relation $\left(\omega^{2}=g k \tanh (k h)\right)$ and radian frequency is related to wave period by $\omega=2 \pi / T$. For monochromatic waves, the wavelength $\lambda=2 \pi / k$ is used in the $G\left(x^{\prime}\right)$ (Eq. 5) width definition (Eq. 4). Convolving the Green's function solution for the linearized extended Boussinesq equations, the W99 $F(y, t)$ source function for monochromatic waves becomes

$$
F(y, t)=D \cos \left(k_{y} y-\omega t\right)
$$


The coefficient $D$ depends upon the wave properties $(a, \omega, \theta)$, and depth $h$ via

$$
D=\frac{2 a\left(\omega^{2}-\alpha_{1} g k(k h)^{3}\right) \cos (\theta)}{\omega I_{1} k\left(1-\alpha(k h)^{2}\right)}
$$

where $\alpha=-0.39$ and $\alpha_{1}=\alpha+1 / 3$ are extended Boussinesq model parameters $(\sqrt[N w o g u]{ }$, 1993) and (W99)

$$
I_{1}=(\pi / \beta)^{1 / 2} \exp \left(-k_{x}^{2} /(4 \beta)\right)
$$

Through (Eq. 10) and $\beta, D$ is also a function of the non-dimensional wavemaker width $\delta$. To satisfy the alongshore periodic boundary conditions, only a finite set of wave angles $\theta_{n}$ are allowed for an alongshore domain length $L_{y}$ such that the alongshore wavenumber $k_{y}=$ $k \sin \left(\theta_{n}\right)=n 2 \pi / L_{y}$, where $n$ is an integer. To generate monochromatic waves, the model inputs are the wave parameters $a_{i}, \omega_{i}, \theta_{i j}$ and $\delta$.

\subsection{Random Directionally-Spread Waves}

The wavemaker can also generate random directional wave fields that are in essence a sum of long-crested waves from multiple directions and frequencies, i.e.,

$$
\eta(x, y, t)=\sum_{i} a_{i} \sum_{j} d_{i j} \cos \left(k_{x}^{(i j)} x+k_{y}^{(i j)} y-\omega_{i} t+\phi_{i j}\right) .
$$

At a particular radian frequency $\omega_{i}$ (where the index $i$ corresponds to frequency) $a_{i}$ is the wave amplitude (in meters), $d_{i j}$ are the directional weights (where index $j$ corresponds to direction) such that

$$
\sum_{j} d_{i j}^{2}=1
$$

and $\phi_{i j}$ is a uniformly distributed random phase for each long-crested wave. For all wave angles $j,\left(k_{x}^{(i j)}\right)^{2}+\left(k_{y}^{(i j)}\right)^{2}=k_{i}^{2}$, where $\left(k_{i}, \omega_{i}\right)$ satisfy the linear surface gravity wave dispersion relation with wave angle,

$$
\theta_{i j}=\tan ^{-1}\left(\frac{k_{y}^{(i j)}}{k_{x}^{(i j)}}\right) .
$$

At each frequency $\omega_{i}$ only directional $j$ wave components that satisfy $k_{y}^{(i j)}=n 2 \pi / L_{y}$ where $n= \pm\{0, \ldots, N\}$ are allowed. The maximum $N$ is chosen so that wave components have incidence angles $\left|\theta_{i N}\right|<50^{\circ}$. W99 suggests that increased evanescent wave modes are generated at large $\theta$ (see 2.1 in W99). In practical nearshore application, highly oblique deep water waves $(k h \gg 1)$ usually will have refracted to smaller angles within this $\pm 50^{\circ}$ range at typical wavemaker depths $(k h \approx 1)$.

To generate random waves, analogous to the monochromatic wave case (Eq. 4), the wavemaker width $W=\bar{\delta} \bar{\lambda} / 2$ where $\bar{\lambda}$ is the mean wavelength and $\bar{\delta}$ represents the bulk nondimensional wavemaker width. With the fixed $\bar{\delta}$ (and $W$ ), the equivalent $\delta$ at each frequency 
will vary. The mean wavelength $\bar{\lambda}=2 \pi / \bar{k}$ depends on the bulk wavenumber $\bar{k}$ derived from the energy weighted mean frequency $\bar{f}$ (Eq. A.2). The cross-shore wavemaker source function $G\left(x^{\prime}\right)$ (Eq. 5) is the same but with $\beta=80(\bar{\delta} \bar{\lambda})^{-2}$.

The alongshore and time-dependent wavemaker source function $F(y, t)$ is defined as

$$
F(y, t)=\sum_{i} D_{i} \sum_{j} d_{i j} \cos \left(k_{y}^{(i j)} y-\omega_{i} t+\phi_{i j}\right)
$$

where the frequency dependent coefficient $D_{i}$ is defined analogously to monochromatic waves,

$$
D_{i}=\frac{2 a_{i}\left(\omega_{i}^{2}-\alpha_{1} g k_{i}\left(k_{i} h\right)^{3}\right) \cos \left(\theta_{2 i}\right)}{\omega_{i} I_{1} k_{i}\left(1-\alpha\left(k_{i} h\right)^{2}\right)}
$$

where $\theta_{2 i}$ is the mean wave angle at frequency $\omega_{i}$ (Kuik et al. 1988), estimated from the prescribed $\theta_{i j}$ and $d_{i j}$ (Appendix). For random waves, the $I_{1}$ definition is similar to that for monochromatic waves (Eq. 10 ) but with $\beta=80(\bar{\delta} \bar{\lambda})^{-2}$ and $\bar{k}_{x}=\bar{k} \cos \left(\bar{\theta}_{2}\right)$, where $\bar{\theta}_{2}$ is the input bulk (energy-weighted) mean wave angle (Eq. A.9. To generate random directional waves, the wavemaker then requires a set of input amplitudes $a_{i}$, frequencies $\omega_{i}$ and directional distribution $d_{i j}$ at all possible $\theta_{i j}$ or $k_{y}^{(i j)}$, which can be directly prescribed.

In many realistic situations, neither the actual incident sea-surface elevation $\eta(x, y, t)$ nor the full frequency-directional spectrum are known. With a pitch-and-roll wave buoy or a colocated pressure sensor and current meter (PUV), only sea-surface elevation spectra $S_{\eta \eta}(f)$ and mean wave angle $\theta_{2}(f)$ (Eq. A.3) and directional spread $\sigma_{\theta}(f)$ (Eq. A.4) based on directional wave moments (e.g., Kuik et al., 1988) can be estimated. Thus, a method to generate waves with statistics matching these input statistics is required. Here, a front-end to the W99 wavemaker is described that takes a set of frequency-dependent input wave statistics $S_{\eta \eta}^{(\mathrm{i})}\left(f_{i}\right), \theta_{2}^{(\mathrm{i})}\left(f_{i}\right)$, $\sigma_{\theta}^{(\mathrm{i})}\left(f_{i}\right)$ at $f_{i}$ and converts them to amplitudes $a_{i}$ and directional weights $d_{i j}$. First spectra are converted to amplitudes

$$
a_{i}^{(\mathrm{i})}=\left[S_{\eta \eta}^{(\mathrm{i})}\left(f_{i}\right) \Delta f\right]^{1 / 2}
$$

At any frequency, directional distribution $d_{i j}$ is given by

$$
d_{i j}^{2}=\exp \left[-\frac{\left(\theta_{i j}-\theta_{2}^{(\mathrm{i})}\left(f_{i}\right)\right)^{2}}{2.07\left(\sigma_{\theta}^{\mathrm{i})}\left(f_{i}\right)\right)^{2}}\right],
$$

at all allowed $k_{y}^{(i j)}$ and subsequently normalized so that $\sum_{j} d_{i j}^{2}=1$. With the directional distribution (Eq. 17), the resulting directional spread (defined in Eq. A.4) can be shown to closely match the input $\sigma_{\theta}^{(\mathrm{i})}$. 

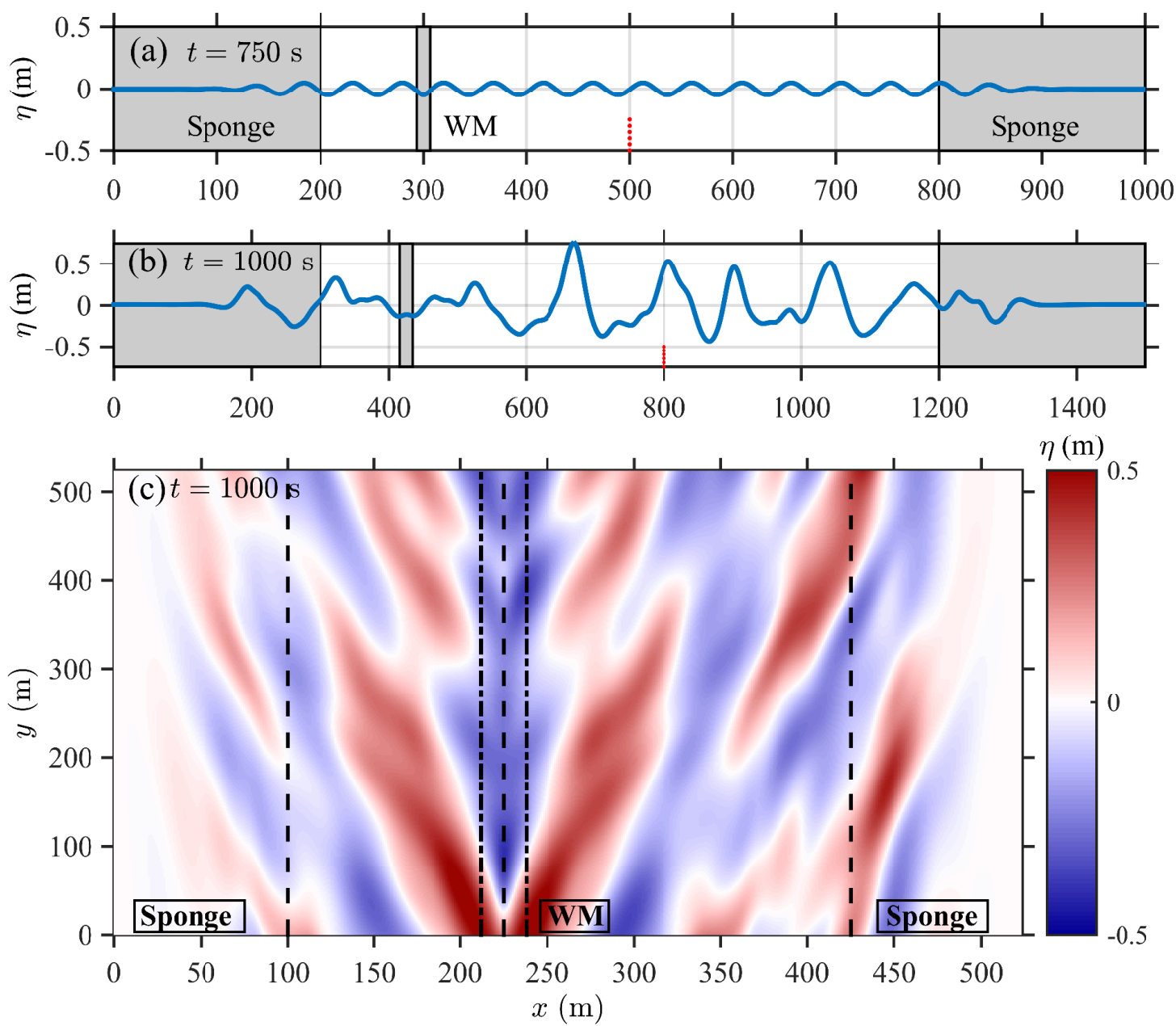

Fig. 1: Snapshots of sea surface elevation $\eta$ versus cross-shore coordinate $x$ for (a) a monochromatic wave with $a / h=0.02$ and $k h=0.775$, (b) random waves with $a / h=0.04$ and $\bar{k} h=0.493$. (c) Sea surface elevation $\eta$ for random obliquely incident directionally-spread waves versus $x$ and alongshore coordinate $y$ for $a / h=0.03$, $\bar{k} h=0.521$, mean wave angle $\theta_{2}=10^{\circ}$, and directional spread $\sigma_{\theta}=5^{\circ}$. In (a) and (b), the light gray regions mark the sponge layers and dark gray regions mark wavemaker location with width (a) $\delta=0.6$ and (b) $\bar{\delta}=0.5$. In panel (c), dashed lines at $x=100 \mathrm{~m}$ and $x=425 \mathrm{~m}$ mark the sponge layers and the region between the two dash-dotted lines at $x=225 \mathrm{~m}$ mark the wavemaker with width $\bar{\delta}=0.5$.

\section{Model Setup}

\subsection{Model Domain}

The wavemaker is tested with two computational domains both with flat bottom of depth $h$ and cross-shore grid resolution $\Delta x=1 \mathrm{~m}$. The first domain is a one-dimensional (1D) channel, akin to a wave flume, for normally incident monochromatic and random wave tests (Fig. 11a,b). The second is a two-dimensional (2D) basin for random directional wave tests (Fig. 17). The 1D channel cross-shore domain length $\left(L_{x}\right)$ ranged from 1000 to $1500 \mathrm{~m}$ with alongshore domain length $L_{y}=10 \mathrm{~m}$ and $\Delta y=1.25 \mathrm{~m}$. The $2 \mathrm{D}$ basin $L_{x}$ varied between 525 and $725 \mathrm{~m}$ with an alongshore domain $L_{y}=1000 \mathrm{~m}$ and $\Delta y=1.33 \mathrm{~m}$. The alongshore boundary conditions are periodic. With the grid resolution, wave dissipation over the domain due to the finite-difference numerics was negligible. All simulations used a $\Delta t=0.02 \mathrm{~s}$ time step. To dissipate wave 
energy and minimize wave reflection (Wei and Kirby, 1995; Wei et al., 1999), frictional sponge layers were placed at the cross-shore boundaries far from the wavemaker with widths ranging from 100 to $400 \mathrm{~m}$, between 1.5 to 5 times the wavelength associated with the peak period (see Fig. 1). Wave energy reflected from the sponge layer was negligible.

\subsection{Monochromatic Waves}

A total of 334 1D channel simulations were conducted with normally-incident $(\theta=0)$ monochromatic waves spanning a range of wave heights $\left(H^{(\mathrm{i})}=2 a^{(\mathrm{i})}\right)$ and periods $\left(T^{(\mathrm{i})}\right)$, water depths, and $\delta$ (Table 1). These simulations did not span a uniformly distributed and independent range of $k h, a / h$, or $\delta$. The nondimensional parameter $a / h$ spans a wide range from very weak nonlinearity $(a / h=0.005)$ to moderately nonlinear $(a / h=0.125)$, with most simulations in the range $0.01<a / h<0.1$. The nondimensional parameter $k h$ spans shallow $(k h=0.14)$ to intermediate $(k h=1.30)$ depth regimes, with most simulations having $k h>0.4$. This $k h$ range is appropriate for the extended Boussinesq equations (Gobbi et al., 2000) and both $a / h$ and $k h$ values are realistic of laboratory or field conditions, and the resulting Ursell number Ur ranges between 0.003-6.2 (Table 1), with most simulations having $\mathrm{Ur}<1$. The $\delta$ range spanned $0.1-1.0$, with most simulations spanning $0.2-0.7$. Simulations were run for $2000 \mathrm{~s}$. At a cross-shore distance of $75-500 \mathrm{~m}$ from the wavemaker, sufficient to reduce evanescent solutions (Wei et al., 1999), a 1000 s sea surface elevation $\eta$ timeseries was extracted. Although the model is nonlinear, model wave height $H^{(\mathrm{m})}$ is computed for an equivalent linear sine-wave of the same $\eta$ variance, i.e.,

$$
H^{(\mathrm{m})}=2 \sqrt{2}\left\langle\eta^{2}\right\rangle^{1 / 2}
$$

where $\eta^{\prime}$ are sea surface fluctuations and \langle\rangle is a time average. Although problematic for large nonlinearity, this allows direct comparison to the input wave height $H^{(\mathrm{i})}$, and is generalizable to random wave spectra. An example monochromatic simulation with $a / h=0.02$ and $k h=$ 0.775 shows the resulting spatial (Fig. 117) and temporal (Fig. 2 a) variability to be sinusoidal and statistically uniform away from the wavemaker and sponge layers.

Table 1: Monochromatic tests $(n=334)$ input parameters: wave height $H^{(\mathrm{i})}$, period $\left.T^{(\mathrm{i})}\right)$, water depth $(h)$, wavemaker width $\delta$, and the resulting nondimensional $a / h, k h$, and Ur.

\begin{tabular}{l|l|l|l|l|l|l}
\hline \hline$H^{(\mathrm{i})}(\mathrm{m})$ & $T^{(\mathrm{i})}(\mathrm{s})$ & $h(\mathrm{~m})$ & $\delta$ & $a / h$ & $k h$ & $\mathrm{Ur}$ \\
\hline $0.1-1$ & $6-20$ & $2-10$ & $0.1-0.9$ & $0.005-$ & $0.142-$ & $0.003-$ \\
& & & & 0.125 & 1.30 & 6.2 \\
\hline
\end{tabular}




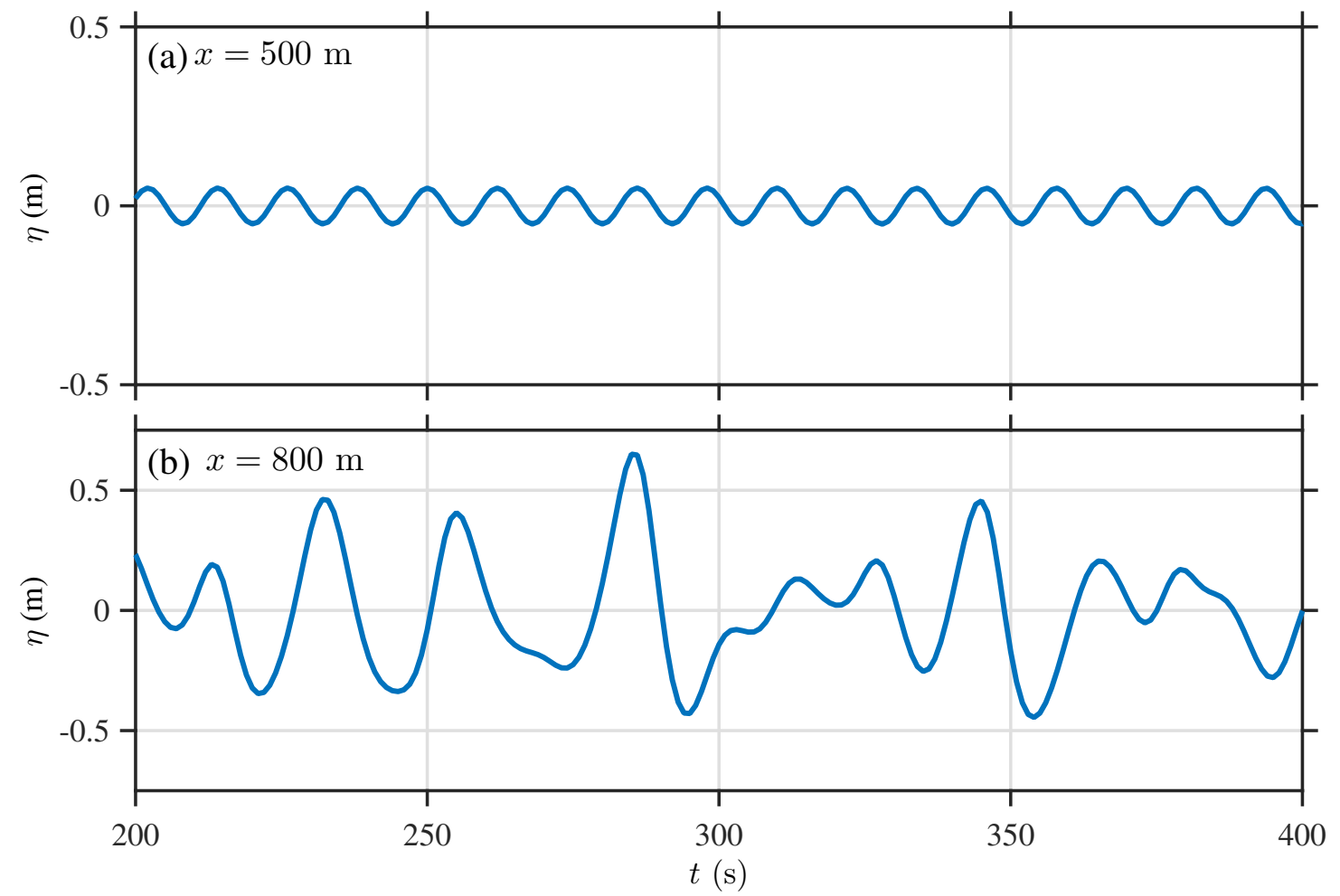

Fig. 2: Water surface elevation $(\eta)$ versus time for (a) monochromatic wave with $a / h=0.02$ and $k h=0.775$ and (b) random waves with $a / h=0.04$ and $\bar{k} h=0.493$. Locations of these time series are denoted as red dotted lines in Fig. 19,b.

\subsection{Random Uni-directional Waves}

The Pierson-Moskowitz (PM) analytic spectrum $S_{\eta \eta}^{\mathrm{PM}}($ Pierson and Moskowitz, 1964), with form

$$
S_{\eta \eta}^{\mathrm{PM}}(f) \propto f^{-5} \exp \left[-\frac{5}{4}\left(\frac{f}{f_{\mathrm{p}}}\right)^{-4}\right],
$$

is used as input spectrum for all random uni-directional simulations. Unlike other more complex analytic spectral forms used in coastal situations, the PM spectrum is chosen for its simplicity and depends on only two parameters; significant wave height $H_{\mathrm{s}}$ and peak period $T_{\mathrm{p}}$ (or peak frequency $f_{\mathrm{p}}=1 / T_{\mathrm{p}}$ ). The input spectrum $S_{\eta \eta}^{(\mathrm{i})}$ is initially set equal to $S_{\eta \eta}^{\mathrm{PM}}$ (Fig. 3). To ensure validity of the extended Boussinesq equations (Gobbi et al., 2000$)$, the input spectrum $S_{\eta \eta}^{(\mathrm{i})}$ is truncated (exponentially brought to zero) at frequencies corresponding to $k h \gtrsim 1.45$ (black dashed curve in Fig. 3). At the low-frequency end, $S_{\eta \eta}^{(\mathrm{i})}$ is cut off at $f=0.04 \mathrm{~Hz}$, where energy is negligible for the chosen $T_{\mathrm{p}}$ range. The input spectrum $S_{\eta \eta}^{(\mathrm{i})}$ is then renormalized so that

$$
\int S_{\eta \eta}^{(\mathrm{i})}(f) d f=\frac{\left(H_{\mathrm{s}}^{(\mathrm{i})}\right)^{2}}{16}
$$

where the integral is over energetic $S_{\eta \eta}^{(\mathrm{i})}$ frequencies, typically the sea-swell frequency band $(0.04-0.3 \mathrm{~Hz})$. For longer period waves $\left(T_{\mathrm{p}}=16 \mathrm{~s}\right)$, the truncated input and PM spectra 


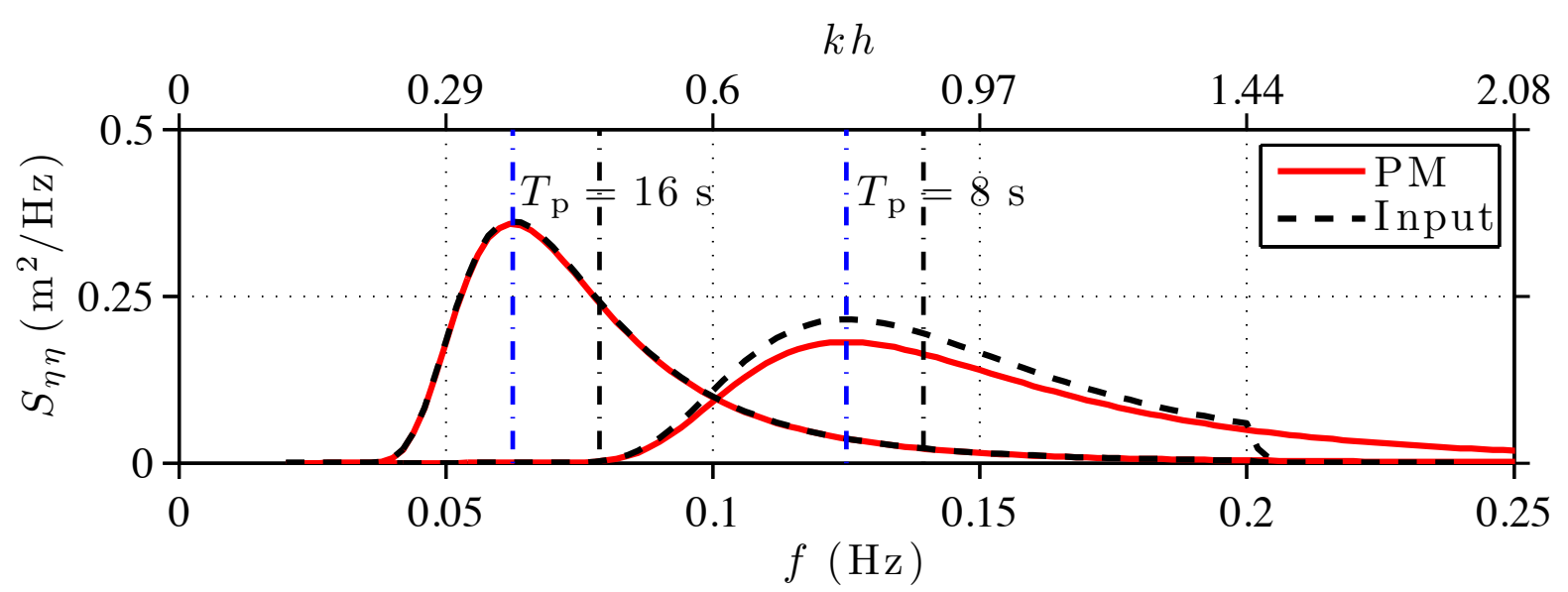

Fig. 3: Analytical Pierson-Moskowitz spectra (red) and model input spectra (dashed black) versus frequency $(f)$ for two peak periods $\left(T_{\mathrm{p}}=16 \mathrm{~s}\right.$ and $\left.T_{\mathrm{p}}=8 \mathrm{~s}\right)$, with significant wave height $H_{\mathrm{s}}=0.5 \mathrm{~m}$, in water depth $h=8 \mathrm{~m}$. The second $x$-axis (top), is the non-dimensional wavenumber corresponding to each frequency in the spectrum. Dotted, dashed lines denote input peak frequency $\left(f_{p}\right.$, blue), and energy-weighted mean frequency $(\bar{f}$, black) of the input spectra, respectively.

are nearly identical, while for shorter period waves $\left(T_{\mathrm{p}}=8 \mathrm{~s}\right)$, deviations between the input and PM spectrum are evident (Fig. 3). The energy-weighted mean frequency $\bar{f}$ (Eq. A.2) and wavenumber $\bar{k}$ are used to characterize each random wave simulation as $\bar{f}$ is a more stable estimate than $f_{p}$. For all spectra, $\bar{f}>f_{\mathrm{p}}$ (see difference between blue and black dotted-dashed lines in Fig. 3).

A total of 36 random, uni-directional (normally-incident $\theta=0^{\circ}$ ) wave simulations were conducted with a range of input $H_{\mathrm{s}}^{(\mathrm{i})}, T_{\mathrm{p}}^{(\mathrm{i})}$ and $h$ (Table 2p. Using the root-mean-square wave amplitude $a=H_{\mathrm{s}} /(2 \sqrt{2})$, the nonlinear parameter $a / h$ range is $0.02-0.06$ (Table 2), valid for the extended Boussinesq equations $([N w o g u, 1993$, Wei et al., 1999). Using the mean wavenumber $\bar{k}$ associated with $\bar{f}$, the dispersion parameter $\bar{k} h$ varies from $0.40-0.95$ (Table 2). Both the $a / h$ and $\bar{k} h$ ranges are appropriate for laboratory or field conditions. These simulations correspond to an Ursell number, defined as $\mathrm{Ur}=(a / h) /(\bar{k} h)^{2}$, between $0.02-0.37$.

For each random uni-directional wave simulation, input amplitudes $a_{i}^{(\mathrm{i})}$ are generated by (Eq. 16) at about 110 discrete equally spaced frequencies $f_{i}=\omega_{i} / 2 \pi$ between $0.04 \mathrm{~Hz}$ and the upper frequency cutoff. Simulations were run for $2000-3000$ s. At a cross-shore distance $>100 \mathrm{~m}$ from the wavemaker, a $1000 \mathrm{~s} \eta$ time series is used to calculate in the sea-swell band $(0.04-0.3 \mathrm{~Hz})$ the modeled frequency spectrum $S_{\eta \eta}^{(\mathrm{m})}, H_{\mathrm{s}}$, and energy-weighted mean frequency $\bar{f}$ to compare with input values. These model statistics are calculated as in realistic nearshore field studies to evaluate the wavemaker's capabilities and limitations in science and engineering applications. An example random wave simulation with $a / h=0.04$ and $\bar{k} h=0.493$ shows the random wave spatial (Fig. 1 b) and temporal (Fig. 2p) variability. At this $\mathrm{Ur}=0.16$, the 
non-zero sea-surface skewness of 0.46 is perceivable.

Table 2: Random uni-directional tests $(n=36)$ input parameters: significant wave height $H_{\mathrm{s}}^{(\mathrm{i})}$, peak period $T_{\mathrm{p}}^{(\mathrm{i})}$, water depth $h$, and the corresponding $a / h, \bar{k} h$, and Ur. In all runs $\bar{\delta}=0.5$.

\begin{tabular}{l|l|l|l|l|l}
\hline \hline$H_{\mathrm{s}}^{(\mathrm{i})}(\mathrm{m})$ & $T_{\mathrm{p}}^{(\mathrm{i})}(\mathrm{s})$ & $h(\mathrm{~m})$ & $a / h$ & $\bar{k} h$ & $\mathrm{Ur}$ \\
\hline $0.5,0.75,1$ & $8,11,16$ & $6-9$ & $0.02-0.06$ & $0.397-0.951$ & $0.02-0.37$ \\
\hline
\end{tabular}

Table 3: Two-dimensional random wave tests $(n=28)$ input parameters: Significant wave height $H_{\mathrm{s}}^{(\mathrm{i})}$, peak period $T_{\mathrm{p}}^{(\mathrm{i})}$, water depth $h$, bulk mean wave angle $\left(\bar{\theta}_{2}^{(\mathrm{i})}\right)$, and bulk directional spread $\left(\bar{\sigma}_{\theta}^{(\mathrm{i})}\right)$, together with corresponding $a / h, \bar{k} h$, and Ur. In all runs, $\bar{\delta}=0.5$.

\begin{tabular}{l|l|l|l|l|l|l|l}
\hline \hline$H_{\mathrm{s}}^{(\mathrm{i})}(\mathrm{m})$ & $T_{\mathrm{p}}^{(\mathrm{i})}(\mathrm{s})$ & $h(\mathrm{~m})$ & $\bar{\theta}_{2}^{(\mathrm{i})}$ & $\bar{\sigma}_{\theta}^{(\mathrm{i})}$ & $a / h$ & $\bar{k} h$ & $\mathrm{Ur}$ \\
\hline $0.4-0.8$ & 8,14 & 8,9 & $0-20$ & $0-20$ & $0.02-$ & $0.521-$ & $0.02-$ \\
& & & & & 0.03 & 0.884 & 0.12 \\
\hline
\end{tabular}

\subsection{Random Directionally Spread Waves}

A total of 28 random directionally-spread wave simulations were performed with the $2 \mathrm{D}$ basin setup across a range of water depths $h, H_{\mathrm{s}}^{(\mathrm{i})}, T_{\mathrm{p}}^{(\mathrm{i})}$, mean wave angle $\bar{\theta}_{2}^{(\mathrm{i})}$, and bulk directional spread $\bar{\sigma}_{\theta}$ (Table 3 ) corresponding to realistic field conditions. The resulting $a / h$ and $\bar{k} h$ range (Table 3) are similar to those for random uni-directional waves (Table 2). A separable frequency-directional wave spectrum $E(\theta, f)=D(\theta) S_{\eta \eta}^{\mathrm{PM}}(f)$ is used with the PM frequency spectrum. The directional spectrum $D(\theta)$ is Gaussian distributed (Eq. 17) and is characterized by a frequency-uniform input mean wave angle $\theta_{2}^{(\mathrm{i})}$ and directional spread $\sigma_{\theta}^{(\mathrm{i})}$. Thus, $\bar{\theta}_{2}^{(\mathrm{i})}=\theta_{2}^{(\mathrm{i})}$ and $\bar{\sigma}_{\theta}^{(\mathrm{i})}=\sigma_{\theta}^{(\mathrm{i})}($ see Section 2.3 .

For each random directionally spread wave simulation, at about 110 discrete equally spaced frequencies $f_{i}=\omega_{i} / 2 \pi$ between $0.04 \mathrm{~Hz}$ and the upper frequency cutoff, input amplitudes $a_{i}$ and $d_{i j}$ are generated by (Eq. 16) and (Eq. 17), respectively. An example snapshot of $\eta$ from a random directionally-spread case with bulk wave angle $\bar{\theta}_{2}^{(\mathrm{i})}=10^{\circ}$ and bulk directional spread $\bar{\sigma}_{\theta}^{(\mathrm{i})}=5^{\circ}$, show irregular waves propagate away from the wavemaker and dissipate at the near-boundary sponge layers (Fig. 1F). Each random directionally-spread simulation was run for $4500 \mathrm{~s}$, with $2000 \mathrm{~s}$ seconds of $\eta$ and horizontal velocity at a location $>100 \mathrm{~m}$ from the wavemaker used to calculate the modeled frequency-dependent spectrum $S_{\eta \eta}^{(\mathrm{m})}(f)$, mean wave angle $\theta_{2}^{(\mathrm{m})}(f)$, and directional-spread $\sigma_{\theta}^{(\mathrm{m})}(f)$ using standard methods (e.g., Kuik et al. 1988; Herbers et al., 1999; Feddersen et al., 2011, see Appendix). The 2000 s modeled time series used for analysis is roughly consistent with the length of time series used to derive these 

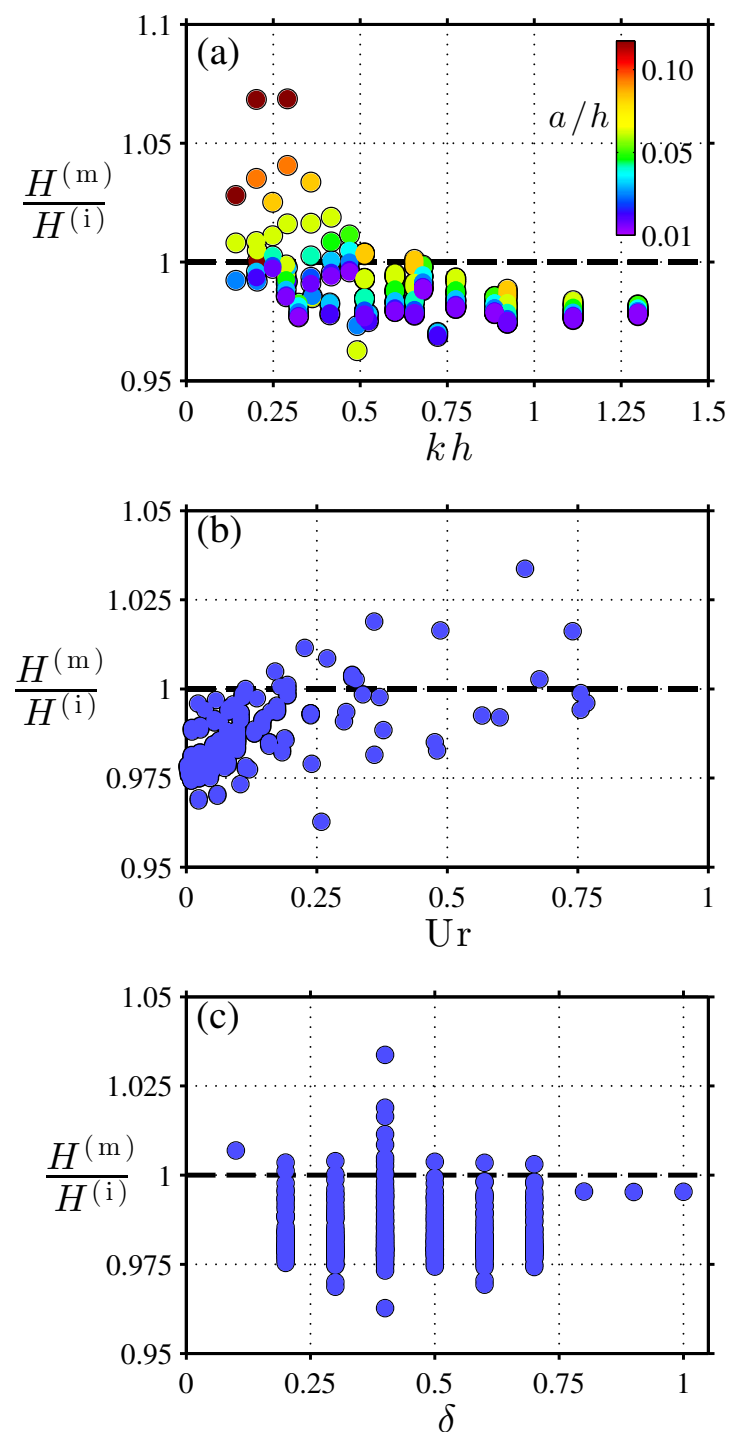

Fig. 4: The ratio of modeled to input wave height $H^{(\mathrm{m})} / H^{(\mathrm{i})}$ versus (a) $k h$ for a range of $a / h$ (see colorbar) and $0.2 \leq \delta \leq 0.7$. (b) $H^{(\mathrm{m})} / H^{(\mathrm{i})}$ versus Ur within the range $0<\mathrm{Ur}<0.1$. (c) $H^{(\mathrm{m})} / H^{(\mathrm{i})}$ versus $\delta$ for a subset of simulations with $a / h \leq 0.1$ and $0.3 \leq k h \leq 1.3$. Single data points for $\delta<0.2$ and $\delta>0.7$ were at constant $k h=0.775$ and $a / h=0.031$.

quantities from field measurements. Overlapped windows and alongshore-averaged spectral estimates result in 100 degrees of freedom for estimating spectra 95\% confidence intervals (Section 4.3). From these, the bulk (energy-weighted) statistics significant wave height $H_{\mathrm{s}}$, mean frequency $\bar{f}$, and bulk mean wave angle $\bar{\theta}_{2}$ and directional spread $\bar{\sigma}_{\theta}$ are calculated and were alongshore uniform.

\section{Results}

\subsection{Monochromatic Waves}

For monochromatic simulations, the ratio of modeled to input wave height $H^{(\mathrm{m})} / H^{(\mathrm{i})}$ was near (within 5\% of) one across almost all $a / h$ and $k h$ and $0.2 \leq \delta \leq 0.7$ (Fig. 4a). This 


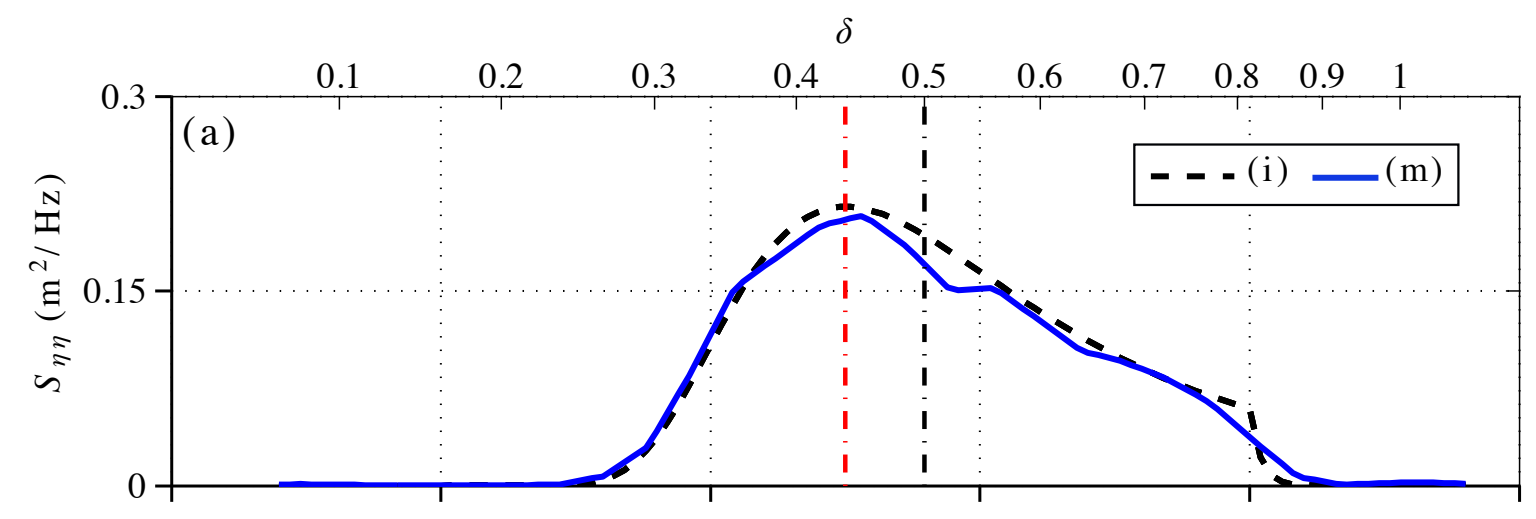

$\delta$

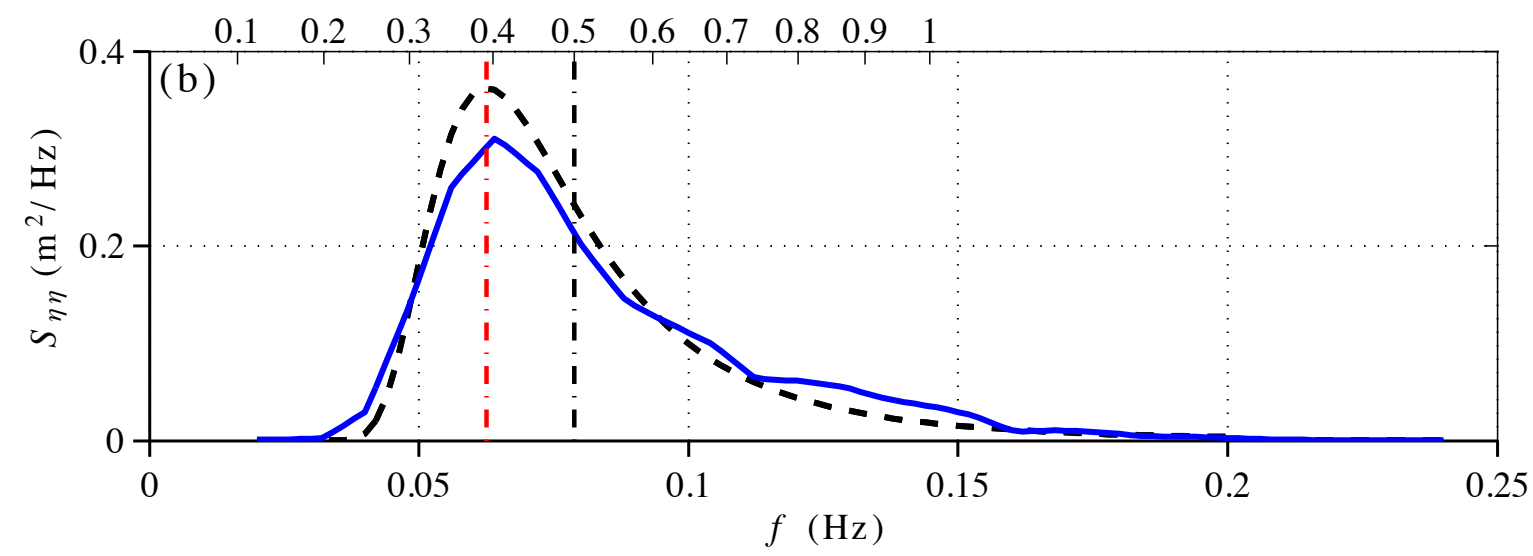

Fig. 5: Random uni-directional case examples: Input (dashed) and modeled (blue) wave spectra $S_{\eta \eta}$ versus (lower) frequency $f$ and (upper) $\delta$ for two cases corresponding to (a) sea $\left(T_{p}=8 \mathrm{~s}\right.$ ) and (b) swell ( $T_{p}=16 \mathrm{~s}$ ) both with $H_{\mathrm{s}}^{(\mathrm{i})}=0.5 \mathrm{~m}, h=8 \mathrm{~m}$, and $a / h=0.02$. In (a) $H_{\mathrm{s}}^{(\mathrm{m})}=0.495 \mathrm{~m}, \bar{f}^{(\mathrm{i})}=0.139 \mathrm{~Hz}, \bar{f}^{(\mathrm{m})}=0.138 \mathrm{~Hz}$, $\bar{k} h^{(\mathrm{i})}=0.88$, and Ur $=0.03$. In (b) $H_{\mathrm{s}}^{(\mathrm{m})}=0.497 \mathrm{~m}, \bar{f}^{(\mathrm{i})}=0.079 \mathrm{~Hz}, \bar{f}^{(\mathrm{m})}=0.083 \mathrm{~Hz}, \bar{k} h^{(\mathrm{i})}=0.46$, and $\mathrm{Ur}=0.1$. Input peak frequency $f_{\mathrm{p}}$ and energy-weighted mean frequency $\bar{f}$ (black dash-dot) are indicated by the vertical red and black dash-dot lines, respectively.

demonstrates that the W99 wavemaker is robust and consistent with the monochromatic linearmodel wave tests of W99. As $k h$ increases, the $H^{(\mathrm{m})} / H^{(\mathrm{i})}$ ratio converges to $\approx 0.98$. At smaller $k h<0.3, H^{(\mathrm{m})} / H^{(\mathrm{i})}$ variability increases with larger nonlinearity $a / h>0.1$ (yellow and red symbols in Fig. 4a). The $a / h$ and $k h$ dependence is collapsed with the Ursell number Ur and shows a weak increase in $H^{(\mathrm{m})} / H^{(\mathrm{i})}$ with Ur (Fig. 4 $\mathrm{b}$ ). The $H^{(\mathrm{m})} / H^{(\mathrm{i})}$ ratio was within $5 \%$ of one for all $\mathrm{Ur}<1$. For $k h \geq 0.3$ and $a / h \leq 0.1$, the ratio $H^{(\mathrm{m})} / H^{(\mathrm{i})}$ had no dependence on the nondimensional wavemaker width $\delta$ (Fig. 4F). Although only a single $k h$ and $a / h$ was simulated for $\delta=0.1$ and $\delta=(0.8,0.9,1.0)$, the wavemaker clearly performs well across a wide $\delta$ range.

\subsection{Random Uni-directional Waves}

For random uni-directional wave cases corresponding to sea $\left(T_{\mathrm{p}}=8 \mathrm{~s}\right)$ and swell $\left(T_{\mathrm{p}}=\right.$ $16 \mathrm{~s})$, both with $H_{\mathrm{s}}^{(\mathrm{i})}=0.5 \mathrm{~m}(a / h=0.02)$, modeled wave spectra $S_{\eta \eta}^{(\mathrm{m})}$ were qualitatively 

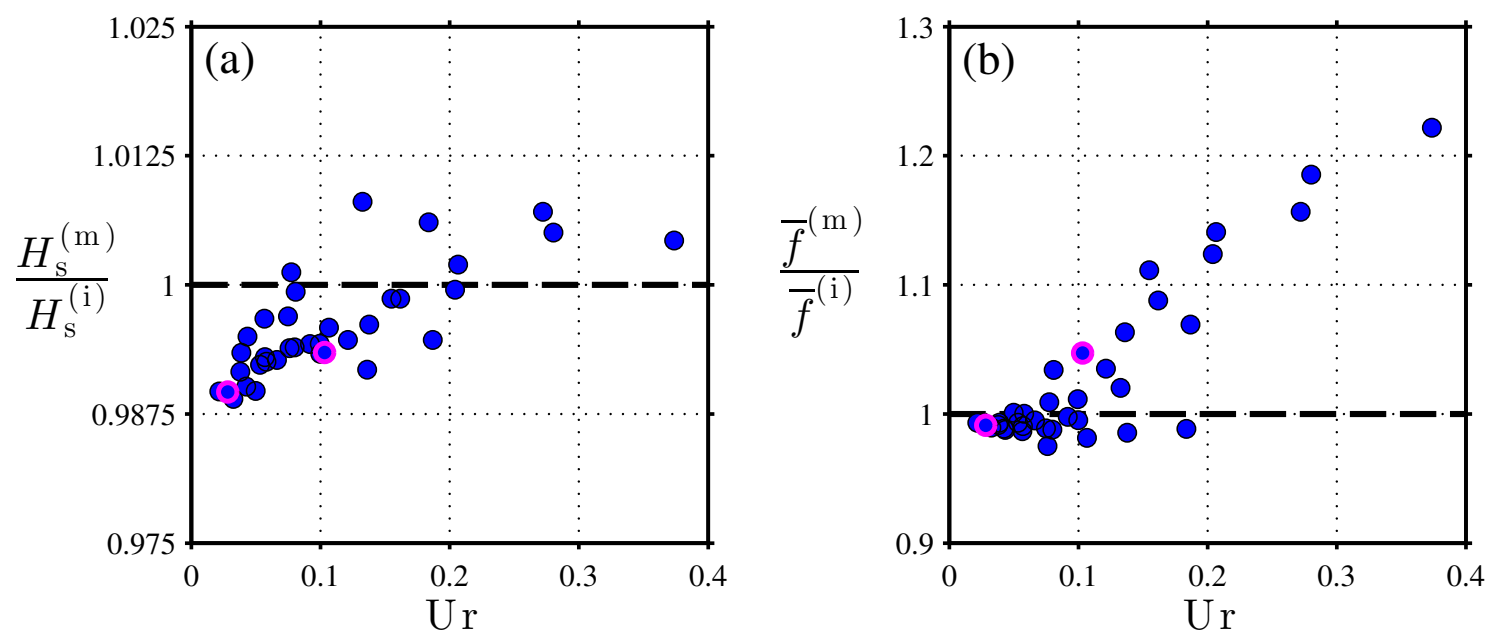

Fig. 6: Ratio of modeled to input (a) significant wave height $H_{\mathrm{s}}^{(\mathrm{m})} / H_{\mathrm{s}}^{(\mathrm{i})}$ and (b) energy-weighted mean frequency $\bar{f}^{(\mathrm{m})} / \bar{f}^{(\mathrm{i})}$ versus Ursell number Ur. The two runs in Fig. 5 are highlighted with magenta.

similar to input spectra $S_{\eta \eta}^{(\mathrm{i})}$ across the range of forced frequencies (Fig. 5). For the sea case $\left(T_{\mathrm{p}}=8 \mathrm{~s}, \bar{k} h=0.88, \mathrm{Ur}=0.03\right), S_{\eta \eta}^{(\mathrm{m})}(f)$ follows $S_{\eta \eta}^{(\mathrm{i})}(f)$ closely (Fig. 5a), resulting in essentially equivalent model and input energy-weighted statistics $\left(H_{\mathrm{s}}^{(\mathrm{m})}=H_{\mathrm{s}}^{(\mathrm{i})}\right.$ and $\bar{f}^{(\mathrm{m})}=$ $\left.\bar{f}^{(\mathrm{i})}\right)$. For the swell case $\left(T_{\mathrm{p}}=16 \mathrm{~s}, \bar{k} h=0.46, \mathrm{Ur}=0.10\right), S_{\eta \eta}^{(\mathrm{m})}$ is weaker than $S_{\eta \eta}^{(\mathrm{i})}$ at $f_{\mathrm{p}}$ and is larger at $\approx 2 f_{\mathrm{p}}$ (Fig. $5 \mathrm{p}$ ), indicating increased nonlinear energy transfer to the harmonic relative to the sea case. Although the resulting $H_{\mathrm{s}}^{(\mathrm{m})}=0.497 \mathrm{~m}$ is very similar to the input $H_{\mathrm{s}}^{(\mathrm{i})}=0.5 \mathrm{~m}$, the transfer of energy to higher harmonics results in $\bar{f}^{(\mathrm{m})}=0.082 \mathrm{~Hz}$, slightly larger than $\bar{f}^{(\mathrm{i})}=0.079 \mathrm{~Hz}$.

In all random waves test cases, the nondimensional wavemaker width $\bar{\delta}=0.5$ where dimensional wavemaker width $W=\bar{\delta} \pi / \bar{k}$ (see Section 2.3). For random waves, there is a range of equivalent $\delta$ corresponding to the range of forced frequencies. To simulate random waves well, the equivalent $\delta$ range must be within the appropriate range as tested for monochromatic waves. The two random uni-directional wave cases mostly span the monochromatic (Fig. 4k) well-simulated $0.1 \leq \delta \leq 1$ range (top axis, Fig. 5). However, for the swell case $\left(T_{\mathrm{p}}=16 \mathrm{~s}\right.$ ), a small part of the variance is generated at $\delta>1$.

After the qualitative $S_{\eta \eta}(f)$ model-input comparison in the sea-swell band, input and modeled bulk statistics, such as significant wave height $H_{\mathrm{s}}$ (Eq. 20) and energy weighted mean frequency $\bar{f}$ (Eq. A.2), are quantitatively compared. As with monochromatic cases, the $a / h$ and $\bar{k} h$ dependence for $H_{\mathrm{s}}^{(\mathrm{m})} / H_{\mathrm{s}}^{(\mathrm{i})}$ and $\bar{f}^{(\mathrm{m})} / \bar{f}^{(\mathrm{i})}$ bulk statistics is collapsed with Ur. The ratio of $H_{\mathrm{s}}^{(\mathrm{m})} / H_{\mathrm{s}}^{(\mathrm{i})}$ was near one ( $<2 \%$ deviation) over the Ur range, increasing weakly with Ur (Fig. 6a) similar to the monochromatic cases (Fig. 4b). Even at $\mathrm{Ur} \approx 0.4$, the wavemaker reproduces the input $H_{\mathrm{s}}^{(\mathrm{i})}$ well, consistent with the two spectral case examples (Fig. 5). For small $\mathrm{Ur}<0.05$, the ratio $\bar{f}^{(\mathrm{m})} / \bar{f}^{(\mathrm{i})} \approx 1$ (Fig. $6 \mathrm{~b}$ ) as nonlinear deviations from the input wave 


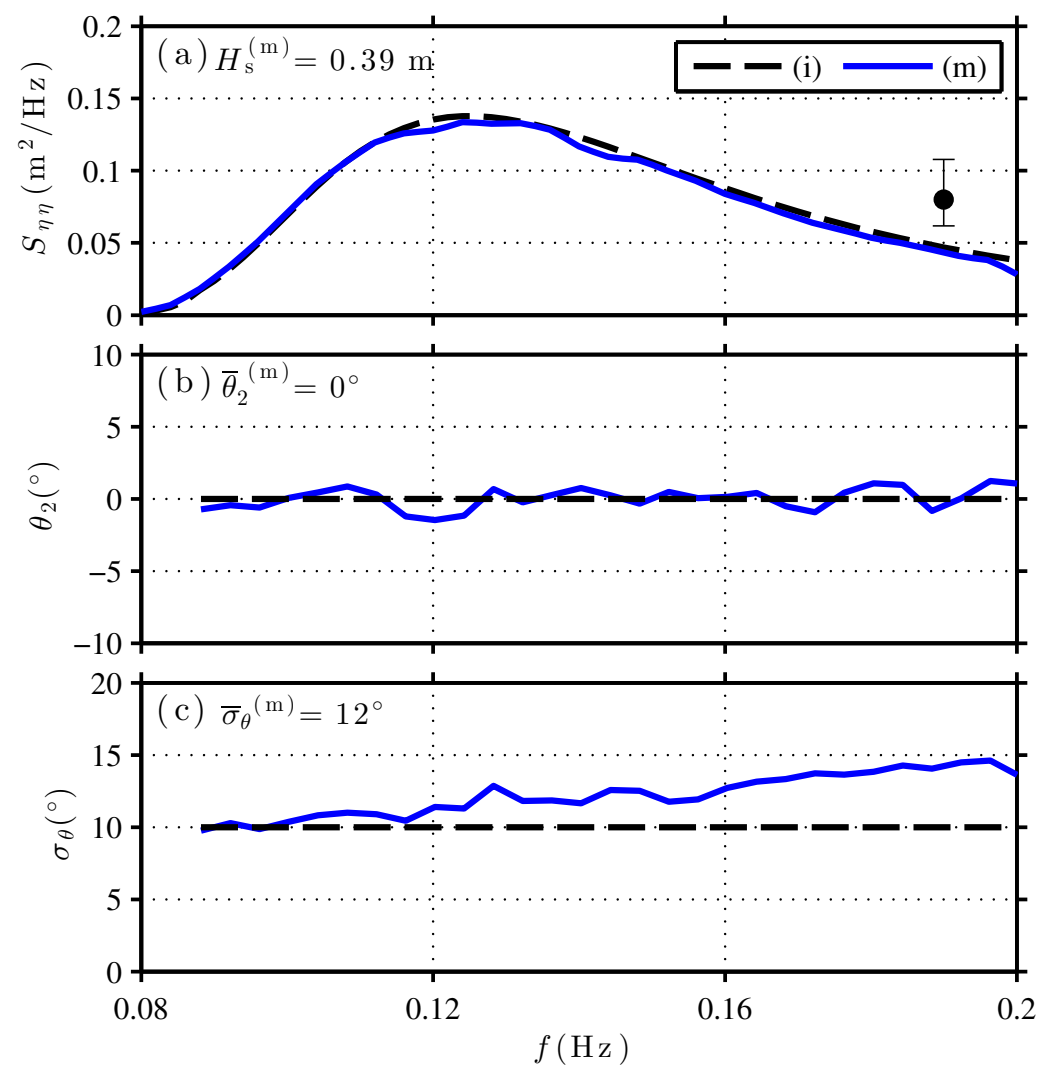

Fig. 7: Random directionally spread wave case example: Input (dashed) and modeled (blue) (a) spectra $S_{\eta \eta}$, (b) mean direction $\theta_{2}$ and (c) directional spread $\left(\sigma_{\theta}\right)$ versus frequency $f$ for $H_{\mathrm{s}}^{(\mathrm{i})}=0.4 \mathrm{~m}, T_{\mathrm{p}}=8 \mathrm{~s}, h=8 \mathrm{~m}$, $a / h=0.02, \bar{k} h=0.88, \mathrm{Ur}=0.02$ and constant $\theta_{2}^{(\mathrm{i})}=0^{\circ}$ and $\sigma_{\theta}^{(\mathrm{i})}=10^{\circ}$. In panels (b) and (c), $\theta_{2}$ and $\sigma_{\theta}$ are shown for $f>0.09 \mathrm{~Hz}$ where $S_{\eta \eta}$ is non-negligible. Spectra $95 \%$ confidence interval at $S_{\eta \eta}=0.08\left(\mathrm{~m}^{2} \mathrm{~Hz}^{-1}\right.$ are noted in (a).

spectra are small. This is consistent with the $T_{\mathrm{p}}=8 \mathrm{~s}$ case example (Fig. 5a) with small $\mathrm{Ur}=0.03$ (magenta dot in Fig. 6b). As Ur increases, $\bar{f}^{(\mathrm{m})} / \bar{f}^{(\mathrm{i})}$ increases linearly to as large as 1.25 for $\mathrm{Ur} \approx 0.4$, consistent with the larger spectral harmonic in the $T_{\mathrm{p}}=16 \mathrm{~s}$ case example Ur $=0.10$ (Fig. 5a). This is similar to the peak frequency ratio for a moving boundary wavemaker for a single random directionally spread case example (Higuera et al. 2013). This $\bar{f}^{(\mathrm{m})} / \bar{f}^{(\mathrm{i})}$ dependence on Ur does not depend on the lower limit of integration $(0.04$ or $0 \mathrm{~Hz})$.

\subsection{Random Directionally Spread Waves}

The wavemaker was tested for random directionally spread waves with input PM spectrum $S_{\eta \eta}^{\mathrm{PM}}(f)$ and a constant mean angle $\theta_{2}^{(\mathrm{i})}(f)$ and directional spread $\sigma_{\theta}^{(\mathrm{i})}(f)$. Two examples are presented with $H_{\mathrm{s}}^{(\mathrm{i})}=0.4 \mathrm{~m}$ and $T_{\mathrm{p}}=8 \mathrm{~s}$, resulting in $a / h=0.02, \bar{k} h=0.88$, and $\mathrm{Ur}=0.02$. The first example has normally incident waves with mean wave angle $\theta_{2}^{(\mathrm{i})}=0^{\circ}$ and the second has obliquely incident waves with $\theta_{2}^{(\mathrm{i})}=10^{\circ}$. Both examples have directional spread $\sigma_{\theta}=$ $10^{\circ}$. For the first case, the modeled spectrum $S_{\eta \eta}^{(\mathrm{m})}$ closely matched the input spectrum $S_{\eta \eta}^{(\mathrm{i})}$ within the confidence intervals at all frequencies (Fig. 77), similar to random uni-directional 


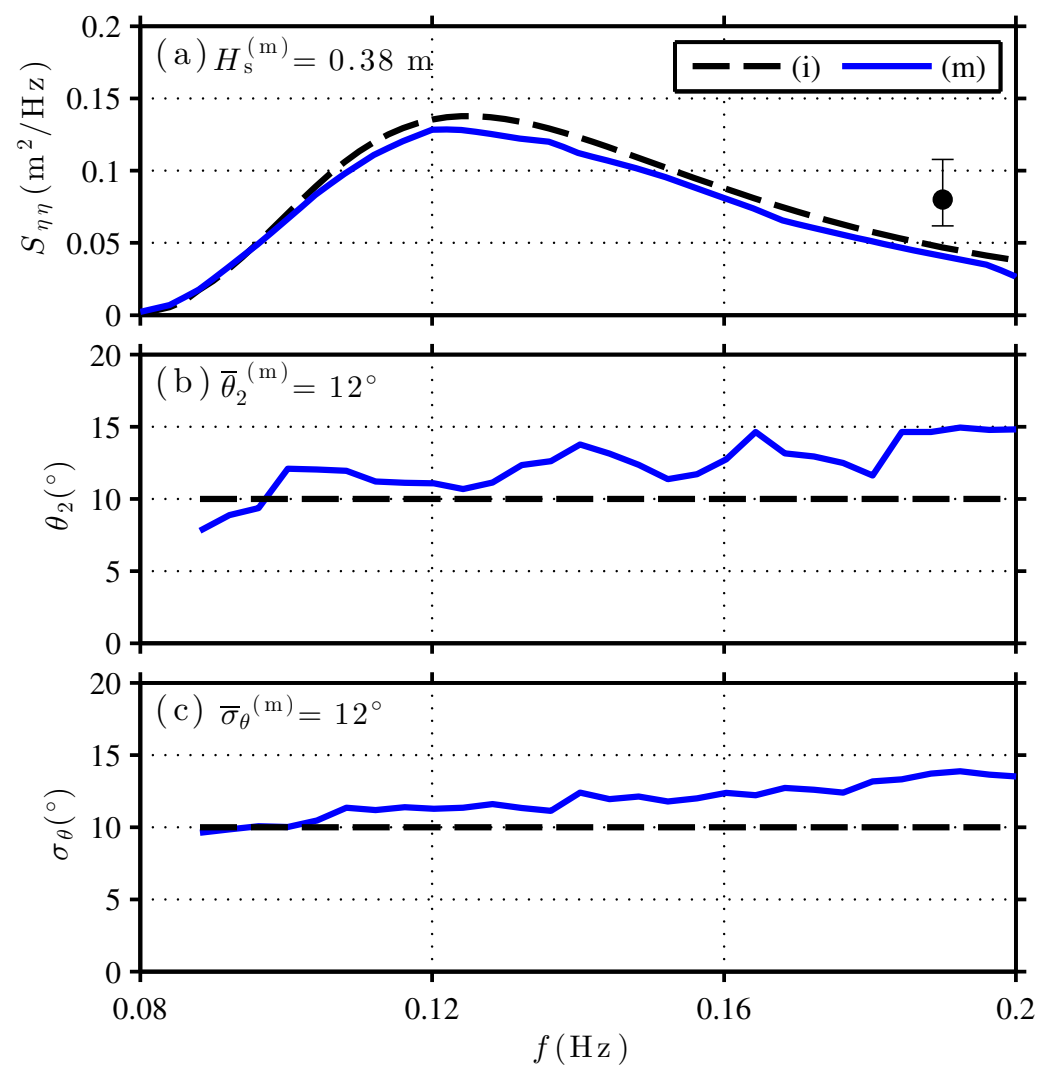

Fig. 8: Random directionally spread case example: Input (dashed) and modeled (blue) (a) spectra $S_{\eta \eta}$, (b) mean wave angle $\theta_{2}$ and (c) directional spread $\sigma_{\theta}$ versus frequency $f$ for $H_{\mathrm{s}}^{(\mathrm{i})}=0.4 \mathrm{~m}, T_{\mathrm{p}}=8 \mathrm{~s}, h=8 \mathrm{~m}, a / h=0.02$, $\bar{k} h=0.88, \mathrm{Ur}=0.02$ and uniform $\theta_{2}^{(\mathrm{i})}=10^{\circ}$ and $\sigma_{\theta}^{(\mathrm{i})}=10^{\circ}$. In panels (b) and (c), $\theta_{2}$ and $\sigma_{\theta}$ are shown for $f>0.09 \mathrm{~Hz}$ where $S_{\eta \eta}$ is non-negligible. Spectra 95\% confidence interval at $S_{\eta \eta}=0.08\left(\mathrm{~m}^{2} \mathrm{~Hz}^{-1}\right.$ are noted in (a).

sea waves at low $a / h$ and moderate $\bar{k} h$ (Fig. 5h). The modeled mean direction $\theta_{2}^{(\mathrm{m})}$ is near zero at all energetic frequencies, closely matching $\theta_{2}^{(\mathrm{i})}$ with deviations from $0^{\circ}$ likely due to crossspectral noise (Fig. 7b). The modeled bulk (energy-weighted) mean wave angle $\bar{\theta}_{2}^{(\mathrm{m})}=0^{\circ}$ also matched the input $\bar{\theta}_{2}^{(\mathrm{i})}=0^{\circ}$. The modeled directional spread $\sigma_{\theta}^{(\mathrm{m})}$ is near (within $2^{\circ}$ of) the input $\sigma_{\theta}^{(\mathrm{i})}=10^{\circ}$ for $f<0.15 \mathrm{~Hz}$ (Fig. 7c), containing the majority of variance. At $f>0.15 \mathrm{~Hz}$, the modeled $\sigma_{\theta}^{(\mathrm{m})}$ is $3^{\circ}-5^{\circ}$ larger than the input $\sigma_{\theta}^{(\mathrm{i})}$ where the spectrum is weaker. This results in modeled bulk (energy-weighted) directional spread $\bar{\sigma}_{\theta}^{(\mathrm{m})}=12^{\circ}$, slightly larger than the input $\bar{\sigma}_{\theta}^{(\mathrm{i})}=10^{\circ}$.

The second case example has an input spectrum $S_{\eta \eta}^{(\mathrm{i})}(f)$ and $\sigma_{\theta}^{(\mathrm{i})}(f)$ as the first case example, but an oblique mean wave angle $\theta_{2}^{(\mathrm{i})}(f)=10^{\circ}$ (Fig. 8 ). As with the first case, $S_{\eta \eta}^{(\mathrm{m})}$ is similar to $S_{\eta \eta}^{(\mathrm{i})}$ within confidence intervals at all frequencies, albeit slightly less near $f_{\mathrm{p}}$, resulting in weaker $H_{\mathrm{s}}^{(\mathrm{m})}=0.38 \mathrm{~m}$ than the input $H_{\mathrm{s}}^{(\mathrm{i})}=0.4 \mathrm{~m}($ Fig. $8 \mathrm{a})$. Note that the ratio $H_{\mathrm{s}}^{(\mathrm{m})} / H_{\mathrm{s}}^{(\mathrm{i})}$ in the random directionally spread examples are slightly less than $H_{\mathrm{s}}^{(\mathrm{m})} / H_{\mathrm{s}}^{(\mathrm{i})}$ in the random unidirectional runs for the same range of Ur. However, $H_{\mathrm{s}}^{(\mathrm{m})} / H_{\mathrm{s}}^{(\mathrm{i})}$ was still within $5 \%$ of one for 

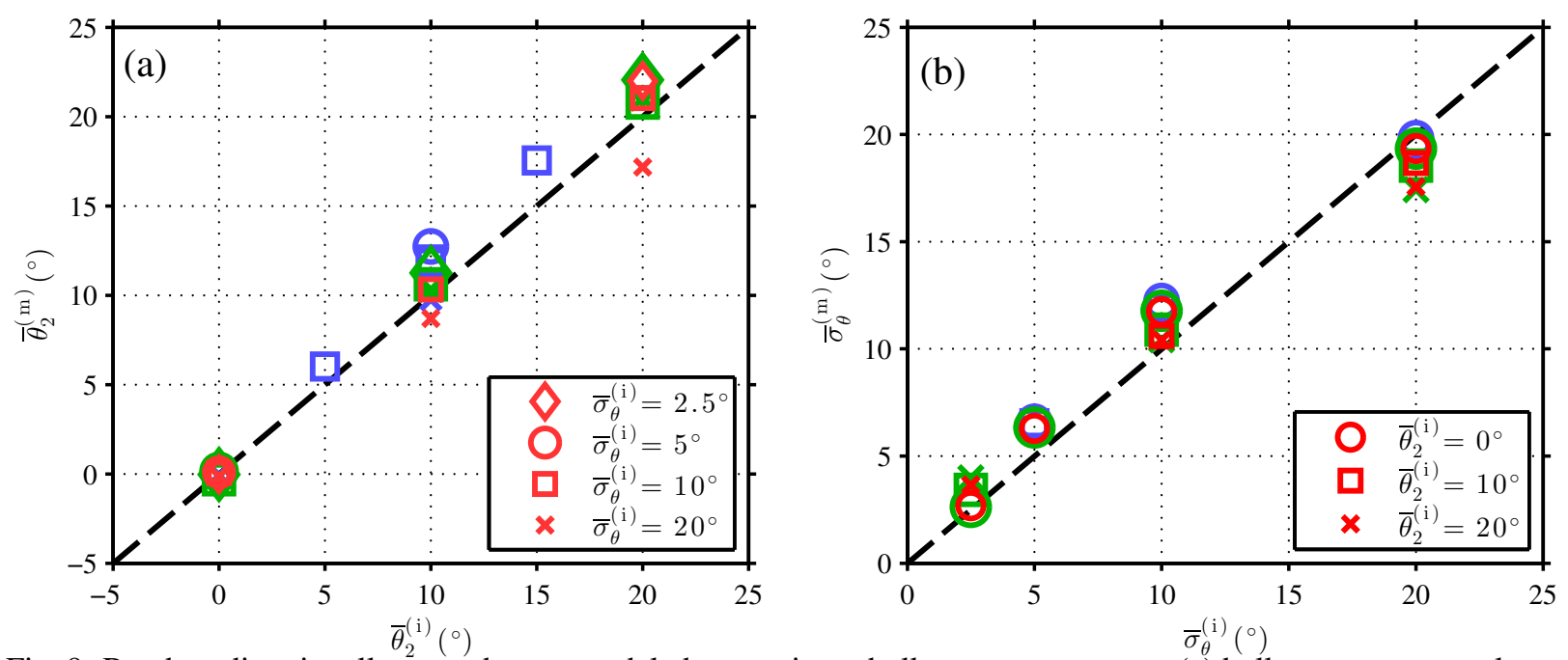

Fig. 9: Random directionally-spread wave modeled versus input bulk wave parameters: (a) bulk mean wave angle $\bar{\theta}_{2}$ for variable $\bar{\sigma}_{\theta}$ and (b) bulk directional spread $\bar{\sigma}_{\theta}$ for variable $\bar{\theta}_{2}$. Symbols are colored by Ur: blue, $\mathrm{Ur}=0.03$; red, $\mathrm{Ur}=0.07$; green, $\mathrm{Ur}=0.12$. Marker sizes are varied for clarity.

all random directionally spread cases. The modeled mean direction $\theta_{2}^{(\mathrm{m})}$ is at or a few degrees larger than the input $\theta_{2}^{(\mathrm{i})}=10^{\circ}$ for $f<0.15 \mathrm{~Hz}$ (Fig. 8 p ). This results in a bulk mean wave angle of $\bar{\theta}_{2}^{(\mathrm{m})}=12^{\circ}$, slightly larger than $\bar{\theta}_{2}^{(\mathrm{i})}=10^{\circ}$. The modeled directional spread $\sigma_{\theta}^{(\mathrm{m})}$ is near (within $2^{\circ}$ of) the input $\sigma_{\theta}^{(\mathrm{i})}=10^{\circ}$ for $f<0.15 \mathrm{~Hz}$ (Fig. $8 \mathrm{c}$ ), and increases to around $\sigma_{\theta}^{(\mathrm{m})}=14^{\circ}$ for $f>0.18 \mathrm{~Hz}$. As with the first case example, the resulting modeled bulk (energy-weighted) directional spread $\bar{\sigma}_{\theta}^{(\mathrm{m})}=12^{\circ}$ is slightly larger than the input $\bar{\sigma}_{\theta}^{(\mathrm{i})}=10^{\circ}$. In both examples and results that follow, the modeled directional coefficients $a_{2}(f)$ and $b_{2}(f)$ (bulk coefficients $\bar{a}_{2}$ and $\left.\bar{b}_{2}\right)$, from which $\theta_{2}(f)$ and $\sigma_{\theta}(f)\left(\bar{\theta}_{2}\right.$ and $\left.\bar{\sigma}_{\theta}\right)$ are derived, are also similar to the input (see Appendix).

The wavemaker's ability to reproduce the desired input bulk directional statistics is further examined with 28 simulations over a range of $\mathrm{Ur}=(0.02,0.07,0.12), \bar{\theta}_{2}$, and $\bar{\sigma}_{\theta}^{(\mathrm{i})}$ (Table 3 ) all based on PM spectra and frequency-uniform $\theta_{2}(f)$ and $\sigma_{\theta}(f)$ (Fig. 9). For all Ur and an input bulk directional spread $2.5^{\circ} \leq \bar{\sigma}_{\theta}^{(\mathrm{i})} \leq 20^{\circ}$, the modeled $\bar{\theta}_{2}^{(\mathrm{m})}$ was near (within $3^{\circ}$ of) the input $\bar{\theta}_{2}^{(\mathrm{i})}$ (Fig. 9 9 ), with $\bar{\theta}_{2}^{(\mathrm{m})}$ biased high on average by $2 \%$. For all Ur and bulk mean angle $0^{\circ} \leq \bar{\theta}_{2}^{(\mathrm{i})} \leq 20^{\circ}$, the modeled $\bar{\sigma}_{\theta}^{(\mathrm{m})}$ is near the input $\bar{\sigma}_{\theta}^{(\mathrm{i})}$ (within $2^{\circ}$ ) with no bias (Fig. $9 \mathrm{~b}$ ). The $\bar{\theta}_{2}^{(\mathrm{m})}$ and the $\bar{\sigma}_{\theta}^{(\mathrm{m})}$ error have no Ur dependence. For non-zero $\bar{\theta}_{2}^{(\mathrm{i})}, \bar{\theta}_{2}^{(\mathrm{m})}$ is reduced at larger directional spread $\bar{\sigma}_{\theta}^{(\mathrm{i})}$ (crosses in Fig. 99). Similarly, for $\bar{\sigma}_{\theta}^{(\mathrm{i})} \geq 10^{\circ}, \bar{\sigma}_{\theta}^{(\mathrm{m})}$ is reduced at larger bulk mean angle $\bar{\theta}_{2}^{(\mathrm{i})}$ (crosses in Fig. 9p). This $\bar{\theta}_{2}^{(\mathrm{m})}$ and $\bar{\sigma}_{\theta}^{(\mathrm{m})}$ reduction is due to the finite $\left(-50^{\circ} \leq \theta \leq 50^{\circ}\right)$ angular region allowed by the wavemaker (Section 2.3 ), potentially resulting in a modified wavemaker directional spectrum and low bias in modeled $\bar{\theta}_{2}$ and $\bar{\sigma}_{\theta}$ at larger $\bar{\theta}_{2}^{\text {(i) }}$ and $\bar{\sigma}_{\theta}^{(\mathrm{i})}$. 


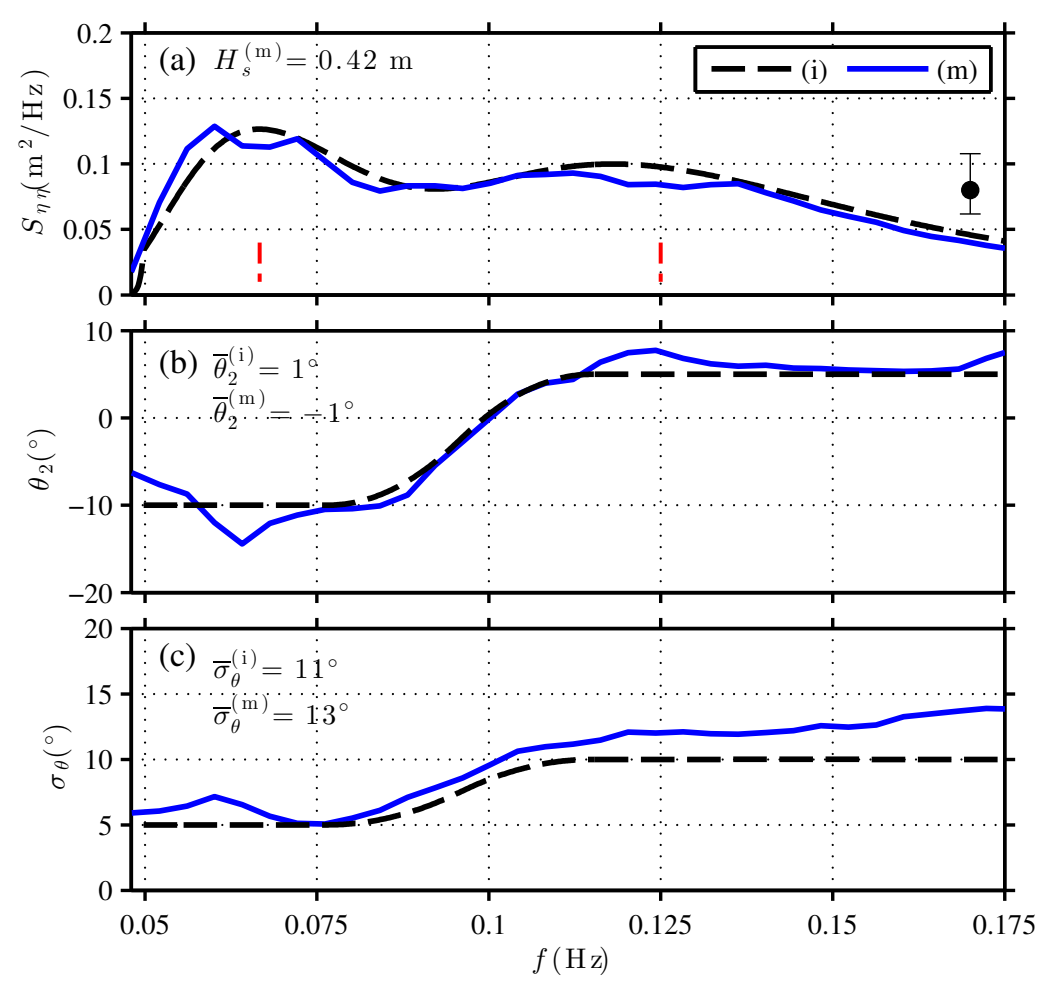

Fig. 10: Random directionally spread opposing sea and swell example: input (black dashed) and modeled (blue) (a) spectra $S_{\eta \eta}$, (b) mean angle $\theta_{2}$ and (c) directional spread $\sigma_{\theta}$ versus frequency $f$. The (swell,sea) PM spectra have $H_{\mathrm{s}}=(0.3,0.3) \mathrm{m}, T_{\mathrm{p}}=(15,8) \mathrm{s}, \theta_{2}=(-10,5)^{\circ}$ and $\sigma_{\theta}=(5,10)^{\circ}$ at $h=8 \mathrm{~m}$ depth. The net $H_{\mathrm{s}}^{(\mathrm{i})}=0.42 \mathrm{~m}$ and $\bar{f}=0.11 \mathrm{~Hz}$. The red vertical tick marks in (a) denote the two input peak frequencies. Spectra $95 \%$ confidence interval at $S_{\eta \eta}=0.08\left(\mathrm{~m}^{2} \mathrm{~Hz}^{-1}\right.$ are noted in (a).

\subsection{Opposing Sea and Swell Case Example}

In the previous random directionally spread cases (Section 4.3), mean wave angle $\theta_{2}(f)$ and directional spread $\sigma_{\theta}(f)$ were uniform with frequency. Here, a final wavemaker test of opposing sea and swell (incident from different quadrants) is conducted with a bimodal sea and swell spectrum with frequency dependent input $\theta_{2}(f)$ and $\sigma_{\theta}(f)$. The swell has a PM spectrum with $H_{\mathrm{s}}=0.3 \mathrm{~m}, T_{\mathrm{p}}=15 \mathrm{~s}$, and frequency-uniform $\theta_{2}(f)=-10^{\circ}$ and $\sigma_{\theta}(f)=5^{\circ}$. The sea also has PM spectrum with $H_{\mathrm{s}}=0.3 \mathrm{~m}, T_{\mathrm{p}}=8 \mathrm{~s}$, and frequency-uniform $\theta_{2}(f)=5^{\circ}$ and $\sigma_{\theta}(f)=10^{\circ}$. The sea and swell frequency spectra are linearly super-imposed, while the directional statistics are smoothly transitioned between $0.086<f<0.105$ to yield the input $S_{\eta \eta}^{(\mathrm{i})}, \theta_{2}^{(\mathrm{i})}(f)$ and $\sigma_{\theta}^{(\mathrm{i})}(f)$ (dashed lines in Fig. 10). The parameters of this opposing sea and swell case are $H_{\mathrm{s}}^{(\mathrm{i})}=0.42 \mathrm{~m}, h=8 \mathrm{~m}$, and $\bar{f}=0.11 \mathrm{~Hz}$, giving $a / h=0.02, \bar{k} h=0.668$, and $\mathrm{Ur}=0.04$.

Similar to the other random wave cases (Figs. 5, 7a, 8a), the modeled spectrum $S_{\eta \eta}^{(\mathrm{m})}$ is similar to the input spectrum $S_{\eta \eta}^{(\mathrm{i})}$ across sea-swell frequencies (Fig. 10 ). The modeled $H_{\mathrm{s}}^{(\mathrm{m})}=$ $0.42 \mathrm{~m}$ and $\bar{f}^{(\mathrm{m})}=0.11 \mathrm{~Hz}$ match the input values, for a $\mathrm{Ur}=0.04$ (e.g., Fig. 6a), consistent with previous results (Figs. 5a, 6). The modeled mean angle $\theta_{2}^{(\mathrm{m})}(f)$ follows the input $\theta_{2}^{(\mathrm{i})}(f)$ 


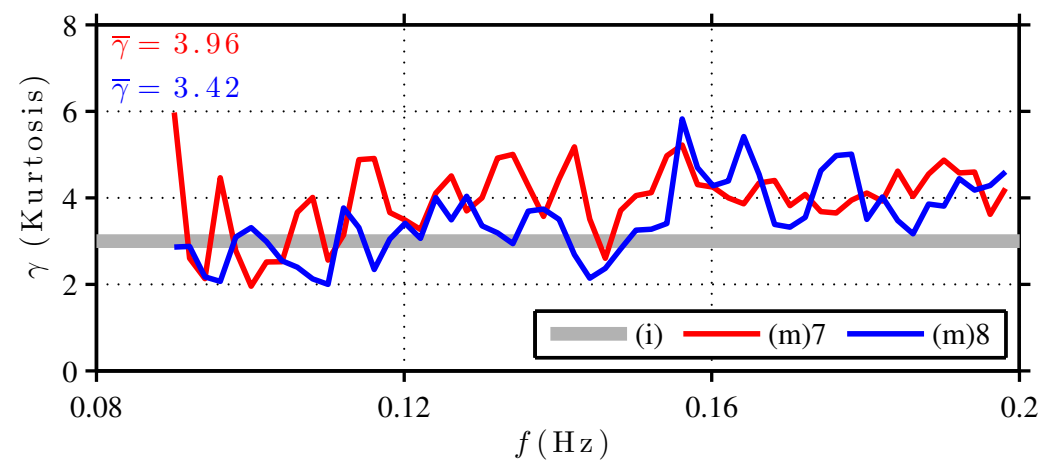

Fig. 11: Kurtosis $\gamma$ (normalized fourth-order directional moment) versus frequency $f$ for two random directionally-spread waves examples $\left(H_{\mathrm{s}}^{(\mathrm{i})}=0.4 \mathrm{~m}, T_{\mathrm{p}}=8 \mathrm{~s}, h=8 \mathrm{~m}, \bar{\sigma}_{\theta}=10^{\circ}\right)$ from Fig. $7\left(\bar{\theta}_{2}=0^{\circ}\right.$, red) and Fig. $8\left(\bar{\theta}_{2}=10^{\circ}\right.$, blue). The theoretical Gaussian directional distribution over the $\pm 50^{\circ}$ aperture is $\gamma=2.95$ (grey).

across sea-swell frequencies transitioning from around $-10^{\circ}$ at $f<0.07 \mathrm{~Hz}$ to $5^{\circ}-8^{\circ}$ for $f>0.1 \mathrm{~Hz}$ (Fig. $10 \mathrm{p}$ ). The resulting bulk mean angle $\bar{\theta}_{2}^{(\mathrm{m})}=-1^{\circ}$ is within $2^{\circ}$ of the input bulk mean angle $\bar{\theta}_{2}^{(\mathrm{i})}=1^{\circ}$, similar to the other cases (Figs. 7b, $8 \mathrm{~b}, 9 \mathrm{a}$ ). The modeled directional spread $\sigma_{\theta}^{(\mathrm{m})}(f)$ also follows the input $\sigma_{\theta}^{(\mathrm{i})}(f)$ across sea-swell frequencies (Fig. 10, ), albeit with high bias as the previous random directionally-spread cases (Fig. 7c, 8c). This bias is greatest, up to $4^{\circ}$ at $f>0.15 \mathrm{~Hz}$. The resulting modeled bulk directional spread $\bar{\sigma}_{\theta}^{(\mathrm{m})}=13^{\circ}$ is slightly larger than $\bar{\sigma}_{\theta}^{(\mathrm{i})}=11^{\circ}$.

\section{Discussion}

Source function (W99) and moving boundary (e.g., Lara et al., 2011) wavemakers have had limited comparison to random directionally spread wave fields. For a single case, the source function wavemaker reproduced the input frequency-directional spectrum $E(\theta, f)$ although with spectral errors and peak direction errors consistent with those reported here (W99). For a single random directionally spread case, a moving boundary wavemaker accurately generated the requested $E(\theta, f)$ although again with errors in frequency and direction similar to those reported here (Higuera et al., 2013). Standard ocean observations (e.g., pitch and roll buoys Kuik et al., 1988) do not provide $E(\theta, f)$ but instead provide bulk directional moments (e.g., $a_{1}(f)$, Appendix). The source function wavemaker has not been previously demonstrated to accurately generate input wave statistics (spectra and directional moments) of a realistic random directionally spread wave field, important to a wide range of science and engineering application.

For example, nearshore circulation and sediment transport studies require that incident wave fields have the appropriate incident radiation stress (e.g., Longuet-Higgins and Stewart, 1964) which depend on the statistics of the frequency and directional spectrum (Battjes, 1972). Given 
the correct incident wave height, mean frequency, bulk mean angle and bulk directional spread, the radiation stress (Feddersen, 2004), wave energy flux, and Stokes drift can be well represented. On alongshore uniform beaches, transient rip current intensity is a strong function of wave height $H_{\mathrm{s}}$ and bulk directional spread $\bar{\sigma}_{\theta}$ (Suanda and Feddersen, 2015) due to finite-crest length wave breaking (Peregrine, 1998). Thus, the W99 wavemaker's ability to generate wave fields which match input statistical properties is important for nearshore application.

In general, the W99 wavemaker generated random wave fields with the correct bulk wave properties $\left(H_{\mathrm{s}}, \bar{f}, \bar{\theta}_{2}, \bar{\sigma}_{\theta}\right)$ for $\mathrm{Ur} \leq 0.12$. This implies that $a / h$ must be small and $\bar{k} h$ must be $O(1)$ at the wavemaker. However, for these model equations $k h<2$ (Gobbi et al., 2000). These constraints, in turn, set limits on wave height $H_{\mathrm{s}}$, wave period $T_{\mathrm{p}}$, and water depth $h$ appropriate for the wavemaker. In common field-based Boussinesq modeling situations (e.g., Chen et al., 2003; Spydell and Feddersen, 2009; Clark et al., 2011; Geiman et al., 2011; Suanda and Feddersen, 2015), waves are generated some distance and depth offshore of the surfzone, where wave nonlinearities are small, and then propagate towards shallow water. Here, these constraints are examined for realistic field usage. For example, for a PM spectra with $H_{\mathrm{s}}=0.8 \mathrm{~m}$ and $T_{\mathrm{p}}=16 \mathrm{~s}$, wavemaker depth $h=4 \mathrm{~m}$ is too shallow $(a / h=0.07, \bar{k} h=0.33, \mathrm{Ur}=0.64)$. However, wavemaker depth $h=10 \mathrm{~m}$ is appropriate $(a / h=0.03, \bar{k} h=0.54, \mathrm{Ur}=0.1)$. Another example with larger waves $H_{\mathrm{s}}=1.5 \mathrm{~m}$ and $T_{\mathrm{p}}=10 \mathrm{~s}$, a wavemaker depth $h=20 \mathrm{~m}$ has too large $\bar{k} h=2.1$. However, wavemaker depth $h=10 \mathrm{~m}$ depth is appropriate $(a / h=0.05, \bar{k} h=1.25, \mathrm{Ur}=0.03)$. This demonstrates that the wavemaker can be used for realistic nearshore applications with an appropriate range of wave height, period, and water depth.

The increased deviation in modeled wave spectra $S_{\eta \eta}$ shape (inferred from $\bar{f}^{(\mathrm{m})} / \bar{f}^{(\mathrm{i})}-$ Fig. 6 ) with increased Ur is not unexpected. The wavemaker theory is based on linearized equations (W99), and the input spectrum is a linear construct where variability is assumed to be independent across frequencies. Reduced spectra at $f_{\mathrm{p}}$ and increased spectra at $2 f_{\mathrm{p}}$ (Fig. 5b) is a result of the weakly nonlinear wave model adapting to the specified frequency dependent forcing. With offshore bispectra boundary conditions stochastic (wave-averaged) Boussinesq models naturally handle this (Herbers and Burton, 1997; Herbers et al., 2003). For Ur $<0.2$, the induced spectral deviations result in $\approx 10 \%$ errors in $\bar{f}$, likely acceptable for studies of wave-averaged processes. However, for detailed nonlinear random wave transformation studies which include energy transfer across frequencies (e.g., Freilich and Guza, 1984; Elgar and Guza, 1985, Elgar et al., 1993), likely smaller wavemaker Ur are required.

The broad range $(0.1 \leq \delta \leq 1)$ over which accurate monochromatic $H^{(\mathrm{m})} / H^{(\mathrm{i})}$ are gener- 
ated (Fig. 4c), gives confidence for generating random waves across a broad range of frequencies with a single $\bar{\delta}$. All random wave simulations used $\bar{\delta}=0.5$, related to the bulk wavenumber $\bar{k}$ and mean frequency $\bar{f}$ (Eq. A.2). However, it is also important that the range of equivalent $\delta$ for an input spectrum (top axis Fig. 5) fall within the validated range. For example, at smaller $\bar{k} h$ (i.e., $\left.T_{p}=18 \mathrm{~s}, h=8 \mathrm{~m}\right)$, the sea-swell band $(0.04-0.3 \mathrm{~Hz})$ spans a larger $\delta$ range $(\delta>1$ at $f=0.14 \mathrm{~Hz}$ ) beyond those tested. At the lower end of the frequency range, $f=0.04 \mathrm{~Hz}$, the equivalent $\delta=0.27$ was well within the validated range and in such cases, a smaller $\bar{\delta}$ should be used.

With periodic alongshore boundary conditions, the wavemaker can only generate a discrete set of wave angles. The alongshore domain width of $L_{y}=1000 \mathrm{~m}$ resulted in relatively accurate modeled $\theta_{2}(f)$ and $\sigma_{\theta}(f)$ as well as bulk $\bar{\theta}_{2}$ and $\bar{\sigma}_{\theta}$ (Section 4.3). These are the quantities that would be measured by a wave buoy or PUV. However, a good $\theta_{2}$ and $\sigma_{\theta}$ modeldata comparison does not ensure that the directional spectrum $D(\theta)$ is well reproduced. The wavemaker's ability to generate a Gaussian $D(\theta)$ is further tested by comparing input and modeled higher order directional moments, skewness $S_{k}(f)$ and kurtosis $\gamma(f)$ (Appendix) for the two random directionally spread cases with $\theta_{2}=\left(0^{\circ}, 10^{\circ}\right)$ (Figs. 7/8). Both input and modeled skewness $S_{k}(f)$ is essentially zero at all $f$ for both normally $\left(\theta_{2}=0^{\circ}\right)$ and obliquely $\left(\theta_{2}=10^{\circ}\right)$ incident waves (not shown). For both cases, the modeled kurtosis $\gamma^{(\mathrm{m})}(f)$ fluctuates between $2-5$, in part due to spectral noise, but is on average slightly larger than the input kurtosis $\gamma^{(\mathrm{i})} \approx 3$ (Fig. 11). For $\bar{\theta}_{2}=\left(0^{\circ}, 10^{\circ}\right)$, the modeled bulk $\bar{\gamma}=(3.4,3.9)$ are somewhat larger than input. This suggests that the wavemaker generated directional spectrum deviates slightly from a Gaussian shape with more energy at higher $\theta$.

Some of the modeled biases may result from the finite set of angles allowed in the domain. For example, with $h=8 \mathrm{~m}$ and $L_{y}=1000 \mathrm{~m}$, the first non-zero wave angle allowed for $T=14 \mathrm{~s}(f=0.071 \mathrm{~Hz})$ is $\pm 7^{\circ}$ whereas for $T=8 \mathrm{~s}(f=0.125 \mathrm{~Hz})$, the first non-zero wave angle allowed is $\pm 4^{\circ}$. Larger $L_{y}$ allow more wave angles. At large $L_{y}=2000 \mathrm{~m}$ and $T=14 \mathrm{~s}$, the first non-zero wave angle is $3.5^{\circ}$. Note, with no mass flux into the alongshore boundaries and adjacent sponge layers, arbitrary $k_{y}$ could be generated. Thus, angle restrictions due to alongshore domain width may result in $\theta_{2}(f)$ and $\sigma_{\theta}(f)$ biases. However, even with these wave angle restrictions, the wavemaker accurately reproduces the $\theta_{2}(f)$ and $\sigma_{\theta}(f)$ over the range of $5^{\circ}$ to $20^{\circ}$ degrees. In addition, highly oblique wave angles are also challenging for the wavemaker, resulting in $\bar{\theta}_{2}$ and $\bar{\sigma}_{\theta}$ bias at large obliquity (Fig. 9). A potential strategy for highly oblique deep water waves could be to provide a refracted input spectrum with reduced obliquity to a wavemaker placed in shallower water. 
Lastly, the model wavemaker does not explicitly generate bound infragravity wave energy. In intermediate water depths that would be associated with the wavemaker, bound infragravity wave energy is observed to be a fraction of the free infragravity energy in field settings (e.g., Okihiro et al., 1992, Herbers et al., 1994). Numerical runup studies using the W99 wavemaker without explicit bound infragravity wave generation simulated well infragravityband shoreline runup compared to video based parameterizations (Guza and Feddersen, 2012). Nevertheless, the generation and evolution of bound and free infragravity wave energy across the entire nearshore to the swashzone is a dense and complex topic (e.g., Herbers et al., 1995, Henderson and Bowen, 2002; de Bakker et al., 2014, and many others). RANS-based modeling of laboratory unidirectional infragravity waves have included 2 nd order bound wave generation (Torres-Freyermuth et al., 2010; Lara et al., 2011; Rijnsdorp et al., 2014). Bound infragravity wave theory for a directionally spread random wave field is more complex. For nearshore infragravity band studies, future work on bound infragravity wave generation with the W99 wavemaker is needed.

\section{Summary}

The W99 source function wavemaker, implemented within the extended nonlinear Boussinesq model funwaveC, was evaluated for its ability to reproduce the sea-swell $(0.04-0.3 \mathrm{~Hz})$ band statistics of input wave fields. Tests were conducted with monochromatic, random unidirectional, and random directionally-spread waves across a range of nondimensional parameters relevant to nearshore environments. For monochromatic waves, the wavemaker accurately reproduced the input wave height $H^{(\mathrm{i})}$ for Ursell number $\operatorname{Ur}=(a / h) /(k h)^{2}<1$, with no dependence upon non-dimensional wavemaker width $0.1<\delta<1$. For random uni-directional waves, the wavemaker reproduced well the input sea surface elevation spectrum $S_{\eta \eta}$ at Ursell number $\mathrm{Ur}=(a / h) /(\bar{k} h)^{2}<0.12$. Frequency-integrated statistics $\left(H_{\mathrm{s}}\right.$, $\bar{f})$ are also well-reproduced for $\mathrm{Ur}<0.2$ with less than $2 \%$ difference between modeled to input significant wave height $H_{\mathrm{s}}$ and $<10 \%$ difference between modeled to input mean frequency $\bar{f}$. For random, directionally spread waves wavemaker-generated frequency dependent $\left(\theta_{2}^{(\mathrm{m})}(f), \sigma_{\theta}^{(\mathrm{m})}(f)\right)$ and bulk $\left(\bar{\theta}_{2}^{(\mathrm{m})}, \bar{\sigma}_{\theta}^{(\mathrm{m})}\right)$ directional statistics were very similar to the input over the range $\left(\bar{\theta}_{2} \leq 20^{\circ} ; \bar{\sigma}_{\theta} \leq 20^{\circ}\right)$ for $\mathrm{Ur} \leq 0.12$.

For accurate sea-swell band random directionally spread wave generation, a number of constraints should be met. First $k h$ must be within the valid Boussinesq model range (here $k h<2$ ). Second, the Ursell number must be relatively small given the $k h$ constraint. Third, the input spectra frequency range should only include equivalent $\delta$ within the validated range. Lastly, two addition directional considerations are recommended: an alongshore domain width 
that allows a range of wave angles to be generated, and input wave angles that are not too obliquely incident. The W99 wavemaker's ability to generate wave fields which match input statistical properties in the sea-swell band demonstrates that it can be used effectively in a range of realistic field-scale nearshore science and engineering applications.

\section{Acknowledgments}

Support was provided by the National Science Foundation (NSF) and the Office of Naval Research (ONR). We thank N. Kumar, R. T. Guza, and M. S. Spydell for helpful discussions on the manuscript. The numerical model, funwaveC, is available online at http : //iod.ucsd.edu/ falk/funwaveC.html. Two anonymous reviewers helped improve the manuscript significantly.

\section{Appendix A. Random Wave Parameters}

The frequency directional spectrum is defined as $S_{\eta \eta}(f) D(\theta ; f)$ where $S_{\eta \eta}$ is the frequency spectrum and $D(\theta ; f)$ is the directional $(\theta)$ distribution at each $f$, defined so that

$$
\int_{-\pi}^{\pi} D(\theta) \mathrm{d} \theta=1
$$

The significant wave height is related to the variance of sea surface fluctuations as:

$$
H_{\mathrm{s}}=4\left[\int_{\mathrm{ss}} S_{\eta \eta} \mathrm{d} f\right]^{1 / 2}
$$

where ss denotes the sea-swell band $(0.04-0.3 \mathrm{~Hz})$. The energy-weighted mean frequency $\bar{f}$ is defined as

$$
\bar{f}=\frac{\int_{\mathrm{ss}} f S_{\eta \eta}(f) \mathrm{d} f}{\int_{\mathrm{ss}} S_{\eta \eta}(f) \mathrm{d} f} .
$$

At each frequency, the directional moments (Kuik et al., 1988)

$$
\begin{aligned}
& a_{n}(f)=\int_{-\pi}^{\pi} \cos (n \theta) D(\theta ; f) \mathrm{d} \theta \\
& b_{n}(f)=\int_{-\pi}^{\pi} \sin (n \theta) D(\theta ; f) \mathrm{d} \theta,
\end{aligned}
$$

where $n=(1,2)$, are estimated from the model $\eta$ and velocity spectra and cross-spectra (e.g., Herbers et al., 1999). The resulting mean wave angle is

$$
\theta_{2}(f)=\frac{1}{2} \arctan \left(\frac{b_{2}(f)}{a_{2}(f)}\right)
$$

and directional spread

$$
\sigma_{\theta}(f)=\frac{1-a_{2}(f) \cos \left(2 \theta_{2}(f)\right)-b_{2}(f) \sin \left(2 \theta_{2}(f)\right)}{2} .
$$


The frequency dependent skewness $S_{k}$ and kurtosis $\gamma$ (normalized third and fourth moments, respectively ) of the directional spectrum are estimated as

$$
S_{k}(f)=\frac{-n_{2}}{\left\{\left(1-m_{2}\right) / 2\right\}^{3 / 2}}
$$

and

$$
\gamma(f)=\frac{6-8 m_{1}+2 m_{2}}{\left\{2\left(1-m_{1}\right)\right\}^{2}}
$$

where

$$
\begin{aligned}
& m_{n}(f)=a_{n}(f) \cos \left(n \theta_{2}\right)+b_{n}(f) \sin \left(n \theta_{2}\right) \\
& n_{n}(f)=b_{n}(f) \cos \left(n \theta_{2}\right)+a_{n}(f) \sin \left(n \theta_{2}\right)
\end{aligned}
$$

as recommended by Kuik et al. (1988). Additionally, energy-weighted directional moments are defined as

$$
\begin{aligned}
& \bar{a}_{n}=\frac{\int_{\mathrm{ss}} a_{n}(f) S_{\eta \eta}(f) \mathrm{d} f}{\int_{\mathrm{ss}} S_{\eta \eta}(f) \mathrm{d} f} \\
& \bar{b}_{n}=\frac{\int_{\mathrm{ss}} b_{n}(f) S_{\eta \eta}(f) \mathrm{d} f}{\int_{\mathrm{ss}} S_{\eta \eta}(f) \mathrm{d} f}
\end{aligned}
$$

giving the bulk (energy-weighted) mean wave angle $\bar{\theta}_{2}$

$$
\bar{\theta}_{2}=\frac{1}{2} \arctan \left(\frac{\bar{b}_{2}}{\bar{a}_{2}}\right) \text {, }
$$

and bulk directional spread $\bar{\sigma}_{\theta}$,

$$
\bar{\sigma}_{\theta}=\frac{1-\bar{a}_{2} \cos \left(2 \bar{\theta}_{2}\right)-\bar{b}_{2} \sin \left(2 \bar{\theta}_{2}\right)}{2}
$$

as well as similarly defined bulk skewness $\bar{S}$ and kurtosis $\bar{\gamma}$.

For the opposing sea and swell case (Section 4.4), the equivalent $\theta_{2}(f)$ and $\sigma_{\theta}(f)$ are calculated as

$$
\begin{aligned}
S_{\eta \eta}(f) & =S_{\eta \eta}^{(\mathrm{sea})}(f)+S_{\eta \eta}^{(\mathrm{sw})}(f) \\
a_{2}(f) & =\frac{a_{2}^{(\mathrm{sea})}(f) S_{\eta \eta}^{(\mathrm{sea})}(f)+a_{2}^{(\mathrm{sw})}(f) S_{\eta \eta}^{(\mathrm{sw})}(f)}{S_{\eta \eta}^{(\mathrm{sea})}(f)+S_{\eta \eta}^{(\mathrm{sw})}(f)} \\
b_{2}(f) & =\frac{b_{2}^{(\mathrm{sea})}(f) S_{\eta \eta}^{(\mathrm{sea})}(f)+b_{2}^{(\mathrm{sw})}(f) S_{\eta \eta}^{(\mathrm{sw})}(f)}{S_{\eta \eta}^{(\mathrm{sea})}(f)+S_{\eta \eta}^{(\mathrm{sw})}(f)}
\end{aligned}
$$

where superscripts (sea) and (sw) indicate the sea and swell components, respectively. The sea and swell directional moments (e.g., $a_{2}^{(\text {sea) }}$ and $a_{2}^{(\mathrm{sw})}$ ) are derived from the prescribed sea and swell $\theta_{2}$ and $\sigma_{\theta}$. From the total $a_{2}$ and $b_{2}$, the bimodal $\theta_{2}(f)$ (Eq. A.3) and $\sigma_{\theta}(f)$ (Eq. A.4) are then estimated.

\section{References}


Battjes, J. A. (1972), Radiation stresses in short-crested waves, J. Mar. Res., 30, 56-64.

Cao, H.-J., and D.-C. Wan (2014), Development of Multidirectional Nonlinear Numerical Wave Tank by NaoeFOAM-SJTU Solver, International Journal of Ocean System Engineering, 4(1), 52-59.

Chawla, A., and J. T. Kirby (2000), A source function method for generation of waves on currents in Boussinesq models, Applied Ocean Research, 22(2), 75-83, doi:10.1016/S0141-1187(00)00005-5.

Chen, Q., R. A. Dalrymple, J. T. Kirby, A. B. Kennedy, and M. C. Haller (1999), Boussinesq modeling of a rip current system, Journal of Geophysical Research: Oceans, 104(C9), 20,617-20,637, doi:10.1029/1999JC900154.

Chen, Q., J. T. Kirby, R. A. Dalrymple, F. Shi, and E. B. Thornton (2003), Boussinesq modeling of longshore currents, Journal of Geophysical Research: Oceans, 108(C11), doi:10.1029/2002JC001308.

Clark, D. B., F. Feddersen, and R. T. Guza (2011), Modeling surf zone tracer plumes: 2. transport and dispersion, Journal of Geophysical Research, 116(C11), C11,028, doi:10.1029/2011JC007211.

de Bakker, A., M. Tissier, and B. Ruessink (2014), Shoreline dissipation of infragravity waves, Continental Shelf Research, 72, 73 - 82, doi:http://dx.doi.org/10.1016/j.csr.2013.11.013.

Elgar, S., and R. Guza (1985), Observations of bispectra of shoaling surface gravity-waves, J. Fluid Mechanics, 161, 425-448, doi: $\{10.1017 /$ S0022112085003007\}.

Elgar, S., R. Guza, and M. Freilich (1993), Observations of nonlinear-interactions in directionally spread shoaling surface gravity-waves, Journal of Geophysical Research-Oceans, 98(C11), 20,299-20,305, doi: $\{10.1029 / 93 \mathrm{JC} 02213\}$.

Feddersen, F. (2004), Effect of wave directional spread on the radiation stress: comparing theory and observations, Coastal Engineering, 51(5-6), 473-481, doi:\{10.1016/j.coastaleng.2004.05.008\}.

Feddersen, F. (2014), The generation of surfzone eddies in a strong alongshore current, Journal of Physical Oceanography, 44(2), 600-617, doi:10.1175/JPO-D-13-051.1.

Feddersen, F., D. B. Clark, and R. T. Guza (2011), Modeling surf zone tracer plumes: 1. waves, mean currents, and low-frequency eddies, Journal of Geophysical Research: Oceans (1978-2012), 116(C11), doi:10.1029/ 2011JC007210.

Freilich, M. H., and R. T. Guza (1984), Nonlinear effects on shoaling surface gravity waves, Philosophical Transactions of the Royal Society of London A: Mathematical, Physical and Engineering Sciences, 311(1515), 1-41, doi:10.1098/rsta.1984.0019.

Geiman, J. D., J. T. Kirby, A. J. H. M. Reniers, and J. H. MacMahan (2011), Effects of wave averaging on estimates of fluid mixing in the surf zone, Journal of Geophysical Research: Oceans, 116(C4), n/a-n/a, doi: 10.1029/2010JC006678, c04006.

Gobbi, M. F., J. T. Kirby, and G. Wei (2000), A fully nonlinear Boussinesq model for surface waves. Part 2. Extension to $O(k h)^{4}$, Journal of Fluid Mechanics, 405, 181-210, doi:10.1017/S0022112099007247.

Guza, R. T., and F. Feddersen (2012), Effect of wave frequency and directional spread on shoreline runup, Geophys. Res. Lett., 39, doi:10.1029/2012GL051959.

Hafsia, Z., M. B. Hadj, H. Lamloumi, and K. Maalel (2009), Internal inlet for wave generation and absorption treatment, Coastal Engineering, 56(9), 951-959.

Henderson, S. M., and A. J. Bowen (2002), Observations of surf beat forcing and dissipation, Journal of Geophysical Research: Oceans, 107(C11), 14-1-14-10, doi:10.1029/2000JC000498, 3193.

Herbers, T., and M. Burton (1997), Nonlinear shoaling of directionally spread waves on a beach, Journal of Geophysical Research-Oceans, 102(C9), 21,101-21,114, doi:\{10.1029/97JC01581\}.

Herbers, T., S. Elgar, and R. T. Guza (1999), Directional spreading of waves in the nearshore, Journal of Geophysical Research-Oceans, 104, 7683-7693, doi:10.1029/1998JC900092.

Herbers, T., M. Orzech, S. Elgar, and R. Guza (2003), Shoaling transformation of wave frequency-directional spectra, Journal of Geophysical Research-Oceans, 108(C1), doi:\{10.1029/2001JC001304\}.

Herbers, T. H. C., S. Elgar, and R. T. Guza (1995), Generation and propagation of infragravity waves, Journal of Geophysical Research: Oceans, 100(C12), 24,863-24,872, doi:10.1029/95JC02680.

Herbers, T. H. C., S. Elgar, and R. T. Guza (1994), Infragravity-frequency (0.005-0.05 Hz) motions on the shelf. Part I: Forced waves, J. Phys. Oceanogr., 24, 917-927, doi:\{10.1175/1520-0485(1994)024〈0917:IFHMOT $\rangle 2$. $0 . \mathrm{CO} ; 2\}$.

Higuera, P., J. L. Lara, and I. J. Losada (2013), Realistic wave generation and active wave absorption for Navier-Stokes models: Application to OpenFOAMß, Coastal Engineering, 71, 102-118, doi:10.1016/j. coastaleng.2012.07.002.

Higuera, P., I. J. Losada, and J. L. Lara (2015), Three-dimensional numerical wave generation with moving boundaries, Coastal Engineering, 101, 35-47, doi:10.1016/j.coastaleng.2015.04.003.

Johnson, D., and C. Pattiaratchi (2006), Boussinesq modelling of transient rip currents, Coastal Engineering, 53(5-6), 419-439, doi:10.1016/j.coastaleng.2005.11.005. 
Kim, G., C. Lee, and K.-D. Suh (2007), Internal generation of waves: Delta source function method and source term addition method, Ocean Engineering, 34(17-18), 2251-2264, doi:10.1016/j.oceaneng.2007.06.002.

Kirby, J. T. (2003), Boussinesq models and applications to nearshore wave propagation, surfzone processes and wave-induced currents, Advances in coastal modeling, 67, 1-41.

Kuik, A. J., G. P. Van Vledder, and L. H. Holthuijsen (1988), A method for the routine analysis of pitch-and-roll buoy wave data, Journal of Physical Oceanography, 18(7), 1020-1034, doi:10.1175/1520-0485(1988)018.

Lara, J. L., N. Garcia, and I. J. Losada (2006), RANS modelling applied to random wave interaction with submerged permeable structures, Coastal Engineering, 53(5-6), 395-417, doi:10.1016/j.coastaleng.2005.11.003.

Lara, J. L., A. Ruju, and I. J. Losada (2011), Reynolds averaged Navier-Stokes modelling of long waves induced by a transient wave group on a beach, Proceedings of the Royal Society of London A: Mathematical, Physical and Engineering Sciences, 467(2129), 1215-1242, doi:10.1098/rspa.2010.0331.

Larsen, J., and H. Dancy (1983), Open boundaries in short wave simulations - a new approach, Coastal Engineering, 7(3), 285-297, doi:10.1016/0378-3839(83)90022-4.

Liam, L. S., D. Adytia, and E. van Groesen (2014), Embedded wave generation for dispersive surface wave models, Ocean Engineering, 80, 73-83, doi:10.1016/j.oceaneng.2014.01.008.

Lin, P., and P. L.-F. Liu (1999), Internal Wave-Maker for Navier-Stokes Equations Models, Journal of Waterway, Port, Coastal, and Ocean Engineering, 125(4), 207-215, doi:10.1061/(ASCE)0733-950X(1999)125:4(207).

Longuet-Higgins, M., and R. Stewart (1964), Radiation stresses in water waves - a physical discussion, with applications, Deep-Sea Research, 11(4), 529-562, doi:\{10.1016/0011-7471(64)90001-4\}.

Madsen, P. A., H. B. Bingham, and H. Liu (2002), A new boussinesq method for fully nonlinear waves from shallow to deep water, Journal of Fluid Mechanics, 462, 1-30, doi:10.1017/S0022112002008467.

Madsen, P. A., H. B. Bingham, and H. A. Schäffer (2003), Boussinesq-type formulations for fully nonlinear and extremely dispersive water waves: derivation and analysis, Proceedings of the Royal Society of London. Series A: Mathematical, Physical and Engineering Sciences, 459(2033), 1075-1104, doi:10.1098/rspa.2002.1067.

Nwogu, O. (1993), Alternative form of boussinesq equations for nearshore wave propagation, Journal of Waterway, Port, Coastal, and Ocean Engineering, 119(6), 618-638, doi:10.1061/(ASCE)0733-950X(1993)119: 6(618).

Okihiro, M., R. T. Guza, and R. J. Seymour (1992), Bound infragravity waves, Journal of Geophysical Research: Oceans, 97(C7), 11,453-11,469, doi:10.1029/92JC00270.

Peregrine, D. H. (1967), Long waves on a beach, Journal of Fluid Mechanics, 27(04), 815-827, doi:10.1017/ S0022112067002605.

Peregrine, D. H. (1998), Surf zone currents, Theoretical and Computational Fluid Dynamics, 10, 295-309, doi: $10.1007 / \mathrm{s} 001620050065$.

Perić, R., and M. Abdel-Maksoud (2015), Generation of free-surface waves by localized source terms in the continuity equation, Ocean Engineering, 109, 567-579, doi:10.1016/j.oceaneng.2015.08.030.

Pierson, W. J., and L. Moskowitz (1964), A proposed spectral form for fully developed wind seas based on the similarity theory of S. A. Kitaigorodskii, Journal of Geophysical Research, 69(24), 5181-5190, doi:10.1029/ JZ069i024p05181.

Rijnsdorp, D. P., P. B. Smit, and M. Zijlema (2014), Non-hydrostatic modelling of infragravity waves under laboratory conditions, Coastal Engineering, 85, 30 - 42, doi:http://dx.doi.org/10.1016/j.coastaleng.2013.11. 011.

Schäffer, H. A., and O. R. Sørensen (2006), On the internal wave generation in Boussinesq and mild-slope equations, Coastal Engineering, 53(4), 319-323, doi:10.1016/j.coastaleng.2005.10.022.

Schäffer, H. A., and G. Klopman (2000), Review of multidirectional active wave absorption methods, Journal of Waterway, Port, Coastal, and Ocean Engineering, 126(2), 88-97, doi:10.1061/(ASCE)0733-950X(2000)126: 2(88).

Shi, F., J. T. Kirby, J. C. Harris, J. D. Geiman, and S. T. Grilli (2012), A high-order adaptive time-stepping TVD solver for Boussinesq modeling of breaking waves and coastal inundation, Ocean Modelling, 43-44, 36-51, doi:10.1016/j.ocemod.2011.12.004.

Spydell, M., and F. Feddersen (2009), Lagrangian drifter dispersion in the surf zone: Directionally spread, normally incident waves, Journal of Physical Oceanography, 39(4), 809-830, doi:10.1175/2008JPO3892.1.

Suanda, S. H., and F. Feddersen (2015), A self-similar scaling for cross-shelf exchange driven by transient rip currents, Geophysical Research Letters, 42(13), 5427-5434, doi:10.1002/2015GL063944.

Torres-Freyermuth, A., J. L. Lara, and I. J. Losada (2010), Numerical modelling of short- and long-wave transformation on a barred beach, Coastal Engineering, 57(3), 317-330, doi:10.1016/j.coastaleng.2009.10.013.

Wei, G., and J. Kirby (1995), Time-dependent numerical code for extended Boussinesq equations, Journal of Waterway, Port, Coastal, and Ocean Engineering, 121(5), 251-261, doi:10.1061/(ASCE)0733-950X(1995) 
121:5(251).

Wei, G., J. T. Kirby, and A. Sinha (1999), Generation of waves in boussinesq models using a source function method, Coastal Engineering, 36(4), 271-299, doi:10.1016/S0378-3839(99)00009-5. 